\title{
Biochemical characterization and inhibition of thermolabile hemolysin from Vibrio parahaemolyticus by phenolic compounds
}

\author{
Luis E Vazquez-Morado ${ }^{1,2}$, Ramon E Robles-Zepeda ${ }^{1}$, Adrian Ochoa-Leyva ${ }^{2}$, Aldo A Arvizu-Flores ${ }^{1}$, Adriana \\ Garibay-Escobar ${ }^{1}$, Francisco Castillo-Yañez ${ }^{1}$, Alonso A Lopez-zavala ${ }^{\text {Corresp. } 1}$ \\ 1 Departamento de Ciencias Quimico Biologicas, Universidad de Sonora, Hermosillo, Sonora, Mexico \\ 2 Departamento de Microbiología Molecular. Instituto de Biotecnología, Universidad Nacional Autónoma de México, Cuernavaca, Morelos, Mexico \\ Corresponding Author: Alonso A Lopez-zavala \\ Email address: alexis.lopez@unison.mx
}

Vibrio parahaemolyticus (Vp), a typical microorganism inhabiting marine ecosystems, uses pathogenic virulence molecules such as hemolysins to cause bacterial infections of both human and marine animals. The thermolabile hemolysin VpTLH lyses human erythrocytes by a phospholipase B/A2 enzymatic activity in egg-yolk lecithin. However, few studies have been characterized the biochemical properties and the use of VpTLH as a molecular target for natural compounds as an alternative to control Vp infection. Here, we evaluated the biochemical and inhibition parameters of the recombinant VpTLH using enzymatic and hemolytic assays and determined the molecular interactions by in silico docking analysis. The highest enzymatic activity was at pH 8 and $50^{\circ} \mathrm{C}$, and it was inactivated by 20 min at $60^{\circ} \mathrm{C}$ with $\mathrm{Tm}=50.9^{\circ} \mathrm{C}$. Additionally, the flavonoids quercetin, epigallocatechin gallate, and morin inhibited the VpTLHactivity with IC50 values of $4.5 \mu \mathrm{M}, 6.3 \mu \mathrm{M}$, and $9.9 \mu \mathrm{M}$, respectively; while phenolics acids were not effective inhibitors for this enzyme. Boltzmann and Arrhenius equation analysis indicate that VpTLH is a thermolabile enzyme. The inhibition of both enzymatic and hemolytic activities by flavonoids agrees with molecular docking, suggesting that flavonoids could interact with the active site's amino acids.

Future research is necessary to evaluate the antibacterial activity of flavonoids against $V p$ in vivo. 
1 Biochemical characterization and inhibition of

2 thermolabile hemolysin from Vibrio parahaemolyticus

3 by phenolic compounds

4

5 Luis E. Vazquez-Morado 1,2, Ramon E. Robles-Zepeda ${ }^{1}$, Adrian Ochoa-Leyva ${ }^{2}$, Aldo A.

6 Arvizu-Flores ${ }^{1}$, Adriana Garibay-Escobar ${ }^{1}$, Francisco J. Castillo-Yañez ${ }^{1}$ and Alonso A.

7 Lopez-Zavala ${ }^{1}$

$8{ }^{1}$ Departamento de Ciencias Químico Biológicas. Universidad de Sonora. Blvd. Rosales y Luis

9 Encinas, C.P. 83000, Hermosillo, Sonora, México.

102 Departamento de Microbiología Molecular, Instituto de Biotecnología, Universidad Nacional

11 Autónoma de México, Avenida Universidad 2001, C.P. 62210, Cuernavaca, Morelos, México

14 Corresponding Author:

15 Alonso A. Lopez-Zavala

16 Blvd. Rosales y Luis Encinas, C.P. 83000, Hermosillo, Sonora, México

17 Email address: alexis.lopez@unison.mx 


\title{
Biochemical characterization and inhibition of thermolabile hemolysin from Vibrio parahaemolyticus by phenolic compounds
}

\author{
Luis E. Vazquez-Morado ${ }^{1,2}$, Ramon E. Robles-Zepeda ${ }^{1}$, Adrian Ochoa-Leyva ${ }^{2}$, Aldo A. \\ Arvizu-Flores ${ }^{1}$, Adriana Garibay-Escobar ${ }^{1}$, Francisco J. Castillo-Yañez ${ }^{1}$ and Alonso A. \\ Lopez-Zavala ${ }^{1}$
}

${ }^{1}$ Departamento de Ciencias Químico Biológicas. Universidad de Sonora. Blvd. Rosales y Luis Encinas, C.P. 83000, Hermosillo, Sonora, México.

2 Departamento de Microbiología Molecular, Instituto de Biotecnología, Universidad Nacional Autónoma de México, Avenida Universidad 2001, C.P. 62210, Cuernavaca, Morelos, México

\section{Corresponding Author:}

Alonso A. Lopez-Zavala

Blvd. Rosales y Luis Encinas, C.P. 83000, Hermosillo, Sonora, México

Email address: alexis.lopez@unison.mx

\section{Abstract}

Vibrio parahaemolyticus ( $\mathrm{Vp}$ ), a typical microorganism inhabiting marine ecosystems, uses pathogenic virulence molecules such as hemolysins to cause bacterial infections of both human and marine animals. The thermolabile hemolysin $V p$ TLH lyses human erythrocytes by a phospholipase B/A2 enzymatic activity in egg-yolk lecithin. However, few studies have been characterized the biochemical properties and the use of $V p$ TLH as a molecular target for natural compounds as an alternative to control $V p$ infection. Here, we evaluated the biochemical and inhibition parameters of the recombinant $V p$ TLH using enzymatic and hemolytic assays and determined the molecular interactions by in silico docking analysis. The highest enzymatic activity was at $\mathrm{pH} 8$ and $50^{\circ} \mathrm{C}$, and it was inactivated by $20 \mathrm{~min}$ at $60^{\circ} \mathrm{C}$ with $\mathrm{Tm}=50.9^{\circ} \mathrm{C}$. Additionally, the flavonoids quercetin, epigallocatechin gallate, and morin inhibited the VpTLH activity with IC50 values of $4.5 \mu \mathrm{M}, 6.3 \mu \mathrm{M}$, and $9.9 \mu \mathrm{M}$, respectively; while phenolics acids were not effective inhibitors for this enzyme. Boltzmann and Arrhenius equation analysis indicate that $V p$ TLH is a thermolabile enzyme. The inhibition of both enzymatic and hemolytic activities by flavonoids agrees with molecular docking, suggesting that flavonoids could interact with the active site's amino acids. Future research is necessary to evaluate the antibacterial activity of flavonoids against $V p$ in vivo.

\section{Introduction}

Vibrio parahaemolyticus $(V p)$ is a Gram-negative bacterium naturally found in marine ecosystems, inhabiting high-valuable species such as fish and shrimps. Recently, $V p$ has been 
57 implicated in high mortalities in shrimp culture ponds, causing significant worldwide economic

58

59

60

61

62

63

64

65

66

67

68

69

70

71

72

73

74

75

76

77

78

79

80

81

82

83

84

85

86

87

88

89

90

91

92

93

94

95

96

losses (Cuéllar-Anjel 2012). Several disease control strategies were assayed in shrimp culture, such as an increased water exchange, phage therapies, probiotics, and supplemented food with prebiotics and antibiotics(Dy et al. 2018; Freire-Moran et al. 2011). The primary treatment for $V p$ 's infections is based on antibiotics use; however, uncontrolled administration interferes with beneficial host-microbiota promoting multidrug bacterial resistance (Santos \& Ramos 2018; Zeng et al. 2019). Thus, it is necessary to develop alternative treatments against $V p$ by identifying novel potential molecular targets ( $\mathrm{Li}$ et al. 2019; Perez-Acosta et al. 2018). Massive sequencing technologies paired with biochemical studies have identified $V p$ virulence elements such as adhesion factors, type III and VI secretion systems, proteases, hemolysins, and others ( $\mathrm{Li}$ et al. 2019)Li et al. 2019).

$V p$ hemolysins are extracellular toxins that lyse the erythrocytes using several molecular mechanisms. Most $V p$ strains express several hemolysins, such as thermostable direct hemolysin (TDH) and TDH-related hemolysin (TRH). These hemolysins have been characterized using structural, biochemical, clinical, and epidemiological approaches (Raghunath 2014; Saito et al. 2015). Both TDH and TRH are pore-forming toxins, while thermolabile hemolysin (TLH) has phospholipase activity (Shinoda et al. 1991)(Shinoda et al. 1991). The th gene encodes the TLH, a full-protein of 418 amino acids $(\mathrm{MW} \approx 47.3 \mathrm{kDa}$ ), and a post-transductional modification removes the $\mathrm{N}$-terminal signal peptide, leaving a mature protein of 399 amino acids (Taniguchi et al. 1986). The TLH from $V p$ " $V p T L H "$ was initially described as a hemolytic factor activated by lecithin, with phospholipase A2 (PLA2)/lysophospholipase enzymatic activity (Shinoda et al. 1991; Yanagase et al. 1970). PLA2 hydrolyzes glycerophospholipids at the sn-2 position to release lysophospholipids, which has a powerful detergent capacity and participates in cell signaling that can induce apoptosis (Flores-Diaz et al. 2016; Murakami \& Kudo 2002). TLH is widely distributed among Vibrio species, such as Vibrio anguillarum, Vibrio vulnificus, Vibrio alginolyticus, Vibrio harveyi, and others (Klein et al. 2014; Wang et al. 2007). TLH research is mainly focused on using this gene as a biomarker to identify virulent strains in epidemiology studies. However, structural and biochemical reports of TLH in comparison with TDH and TRH are scarce. Thermolabile hemolysins from $V$. harveyi and $V$. alginolyticus (VhTLH and VaTLH, respectively) were virulence factors against fish (Jia et al. 2010; Zhong et al. 2006).V. vulnificus $\mathrm{TLH}(V v \mathrm{TLH})$ lost both enzymatic and hemolytic activity after 30 minutes at $55^{\circ} \mathrm{C}$ and $65^{\circ} \mathrm{C}$, respectively. This enzyme also showed cytotoxic activity against flounder gills cells and in vivo toxicity with a medium lethal dose of $1.2 \mu \mathrm{g}$ of protein per gram of flounder (Zhong et al. 2006). Recombinant $\mathrm{VaTLH}$ was also toxic when injected into zebrafish with a lethal dose $(0.8 \mu \mathrm{g} / \mathrm{gr})$ fish). Li et al. (2013) reported that Vibrio alguillarum secretes a TLH with potent hemolytic specific activity against rainbow trout erythrocytes ( $\mathrm{Li}$ et al. 2013).

TLH has a high-conserved amino acid sequence ( $>90 \%)$ among Vibrio species and contains a characteristic GDSL motif belonging to the esterase-lipases family (Akoh et al. 2004). This motif is located in the C-terminal domain (residues 151-406) of the $\mathrm{VpTLH}$ sequence (Taniguchi et al. 1986). TLHs belong to the serine-proteases family and contain a catalytic triad composed of

Peer) reviewing PDF | (2020:09:52773:1:0:CHECK 6 Nov 2020) 
97 serine 153, aspartic acid 154, and histidine 393 (numbering is according to the $V p$ TLH

98 sequence), which is located in the SGNH hydrolases domain (Carter \& Wells 1988; Taniguchi et al. 1986). The SGNH domain comprises four conserved blocks, I, II, III, and V, based on many esterases/hydrolases enzyme's catalytic mechanism. Briefly, block-I comprises the typical GXSXG motif found in lipases/esterases, in which Ser153 acts as the nucleophile during catalysis. While glycine 204 and asparagine 248 in blocks II and III are the proton donors in the oxyanion cavity. On the other hand, the histidine 393 located in block V activates the catalytic residue Ser153, and the aspartic acid 390 stabilizes the tetrahedral intermediate, ensuring the correct orientation during catalysis (Akoh et al. 2004; Upton \& Buckley 1995; Wan et al. 2019). Plants mainly synthesized the phenolic compounds as secondary metabolites, such as phenolic acids (caffeic acid, gallic acid, among others) and polyphenols (p.e. flavonoids: quercetin, rutin, morin, among others) (Cheynier 2012; Panche et al. 2016). These compounds function as antioxidants, cytotoxic, antifungal, antibacterial, and enzyme inhibitors destabilizing the cell membrane (Özçelik et al. 2011; Petrescu et al. 2019).

The quercetin inhibits the enzymatic activity of PLA2 of the snake Crotalus durissus terrificus venom via hydrogen bonds and hydrophobic interactions with the enzyme active site (Cotrim et al. 2011). Furthermore, morin and rutin were potent PLAs inhibitors of Crotalus atrox and Crotalus durissus cascavella, respectively; but rutin also inhibits PLA2 from the porcine pancreas (Iglesias et al. 2005; Lindahl \& Tagesson 1997). Other phenolics compounds as gallic, ferulic, caffeic acids, and epigallocatechin gallate inhibited both enzymatic activity and cytotoxic activity Crotalus durissus cumanensis PLA2 (Pereanez et al. 2011). VpTLH displays PLA2 enzymatic activity and has similar active site amino acids (serine, histidine, and glutamic/aspartic acid) found in several venom snake PLA2 (Murakami \& Kudo 2002). Zhao et al., (2020), reported that resveratrol (stilbene group of polyphenols) efficiently inhibits VhTLH hemolytic activity and cytotoxicity directly by binding to the active site. However, a high resveratrol dose shows tissue accumulation in fish (Takifugu rubripes), resulting in toxic effects (Zhao et al. 2020). Therefore, additional research will provide information about the potential use of phenolic compounds derivatives to inhibit pathogenic factors as TLH.

As previously mentioned, most studies have focused on TDH and TRH and its role in human infections by $V$. parahaemolyticus. However, only a few studies have characterized the biochemical properties of $V p T L H$, and there are not reported enzyme inhibitors. Contrary, phenolic compounds are effective inhibitors against several PLA2 and VhTLH. Therefore, these compounds could also inhibit $V p$ TLH activity providing novel alternatives for treating $V p$ infections. In this work, the effect of $\mathrm{pH}$ and temperature on the enzymatic activity of recombinant $V p$ TLH was evaluated, and the kinetics parameters were determined using $p$ nitrophenyl laurate (PNPL) as substrate. Additionally, we also analyzed several phenolics compounds as inhibitors of both enzymatic and hemolytic activity, and we describe the possible molecular interactions using in silico molecular docking. 


\section{Materials \& Methods}

138

139

140

141

142

143

144

145

146

147

148

149

150

151

152

153

154

155

156

157

158

159

160

161

162

163

164

165

166

167

168

169

170

171

172

173

174

175

176

All reagents were ACS, electrophoresis, or molecular biology grade as required and were purchased in Merck (Sigma-Aldrich). Exceptions were indicated in the text.

\section{Cloning the $V p$ TLH gene and recombinant protein expression.}

The nucleotide sequence of $V p$ TLH used in this study was obtained from the gene bank accession number AB012596.1. $\mathrm{VpTLH}$ was obtained as a synthetic gene (Atom ${ }^{\circledR}$ ) and cloned into pET-28b (+) plasmid, adding the C-terminal $6 \mathrm{x}$-His tag for the purification process. Chemically competent E. coli BL-21 strain rosetta II cells were transformed with $V p$ TLH plasmid by thermal shock and incubated in SOC media (tryptone $2 \% \mathrm{w} / \mathrm{v}$, yeast extract $0.5 \%$ w/v, $10 \mathrm{mM} \mathrm{NaCl}, 2.5 \mathrm{mM} \mathrm{KCl}, 10 \mathrm{mM} \mathrm{MgCl} 2,10 \mathrm{mM} \mathrm{MgSO} 4$ and $20 \mathrm{mM}$ glucose) at $37^{\circ} \mathrm{C}$ by $4 \mathrm{~h}$. Bacterial cells were plated in Luria-Bertani agar plates supplemented with kanamycin ( 25 $\mu \mathrm{g} / \mathrm{ml}$ ) at $37^{\circ} \mathrm{C}$ overnight for the plasmid selection. After that, a single colony was inoculated in $5 \mathrm{ml}$ of the antibiotic-LB medium by four h at room temperature; this culture was scaled up to 50 $\mathrm{ml}$ under the same conditions and incubated overnight. Subsequently, a Fernbach flask containing 11 of LB medium added with kanamycin $(25 \mu \mathrm{g} / \mathrm{ml})$ was inoculated with the $50 \mathrm{ml}$ culture and incubated at $37^{\circ} \mathrm{C}$ and $220 \mathrm{rpm}$. When the optical density reached $\approx 0.6$ units $(\lambda=600$ $\mathrm{nm}$ ), we added IPTG (Isopropyl $\beta$-D-1-thiogalactopyranoside) to a final concentration of $1 \mathrm{mM}$, inducing the overexpression of $V p$ TLH. The Fernbach flask was maintained in an orbital shaker (200 rpm) for 16 hours at $25^{\circ} \mathrm{C}$. Bacterial cells were recovered by centrifugation at 7,000 rpm for 20 minutes at $4{ }^{\circ} \mathrm{C}$, and the pellet was washed using $0.7 \% \mathrm{NaCl}$ and spun as before. The supernatant was discarded, and the bacterial cell pellet was stored at $-80^{\circ} \mathrm{C}$ until use.

\section{Protein purification and in vitro refolding.}

$V p$ TLH was recovered from frozen pellet, which was resuspended in lysis buffer $(50 \mathrm{mM}$ Tris base, $1 \mathrm{mM}$ DTT, $5 \mathrm{mM}$ Benzamidine, $5 \mathrm{mM}$ EDTA, $100 \mathrm{mM} \mathrm{NaCl} ; \mathrm{pH} 7.0)$ at ratio 1:8 (w/v). Bacterial cells were lysed by sonication on an ice bed with six pulses of 5 -s and 5 -s rest at $30 \%$ amplitude. The homogenate was clarified at $12,000 \mathrm{rpm}$ for $20 \mathrm{~min}$ at $4^{\circ} \mathrm{C}$, and SDS-PAGE (12\%) stained with blue-coomassie was used to analyze the protein expression in soluble and insoluble fractions (Laemmli 1970). Several overexpression conditions were analyzed, but in all cases, $V p$ TLH was obtained as inclusion bodies. Therefore, inclusion bodies were isolated from insoluble cellular debris; which was resuspended by sonication (as before) using buffer 1 (50 mM Tris base, DTT $1 \mathrm{mM}, 5 \mathrm{mM}$ EDTA, $2 \%$ Triton $\mathrm{x}-100 ; \mathrm{pH} 7.0)$ at ratio $1: 4(\mathrm{w} / \mathrm{v})$, and the homogenate was centrifugated at $12,000 \mathrm{rpm}$ for $20 \mathrm{~min}$. The precipitate was recovered, and centrifugation was repeated three times. After that, the pellet was 2-times washed in buffer 2 (buffer 1, without Triton X-100) under the same conditions. Finally, the recovered inclusion bodies were solubilized using urea $(50 \mathrm{mM}$ Tris base, DTT $1 \mathrm{mM}$, urea $8 \mathrm{M}, \mathrm{pH} 7.0)$ by sonication and incubated overnight at $4^{\circ} \mathrm{C}$ with constant stirring. The homogenate was clarified $\left(12 ; 000 \mathrm{rpm}\right.$ at $4^{\circ} \mathrm{C}$ for $\left.30 \mathrm{~min}\right)$, and the soluble fraction containing the $\mathrm{VpTLH}$ in urea $8 \mathrm{M}$ was 
177

178

179

180

181

182

183

184

185

186

187

188

189

190

191

192

193

194

195

196

197

198

199

200

201

202

203

204

205

206

207

208

209

210

211

212

213

214

215

216

recovered. The protein concentration was quantified at $280 \mathrm{~nm}$ in a nanodrop ${ }^{\circledR}$ equipment $(\varepsilon \approx$ 96,510 M-1 cm-1). VpTLH was purified by Immobilized Nickel affinity chromatography on an Äkta Prime (GE) under denaturing conditions at $25^{\circ} \mathrm{C}$. Therefore all buffers used during this process contained $8 \mathrm{M}$ urea. Briefly, $1 \mathrm{ml}$ HiTrap ${ }^{\circledR}$ IMAC-HP column was equilibrated with five volumes of buffer $\mathrm{A}$ ( $50 \mathrm{mM}$ Tris base, $500 \mathrm{mM} \mathrm{NaCl}, 8 \mathrm{M}$ urea; $\mathrm{pH} 7.4$ ), then $4 \mathrm{ml}$ (15 mg denatured protein) of $V p$ TLH solution was loaded into the column. Non-bounded proteins were washed with buffer $A$ until absorbance $(\lambda=280 \mathrm{~nm})$ reached the base-line. Bound proteins were eluted with a linear gradient of buffer B (buffer A $+500 \mathrm{mM}$ imidazole), eluted fractions were collected, and SDS-PAGE analyzed. Fractions containing a single band with a molecular weight of $48.3 \mathrm{kDa}$ were pooled and refolded by dialysis with a cut-off membrane of $12-14 \mathrm{kDa}$ at $4^{\circ} \mathrm{C}$. Refolding was carrying out by sequentially decreasing urea concentration $(4,1 \mathrm{M}$ and without urea) in buffer $50 \mathrm{mM}$ Tris-Base buffer at $\mathrm{pH} 7.5$ (250 $\mathrm{mL}$ by 6 hours each). Buffer without a chaotropic agent was changed twice (12 hours each), then the protein solution was removed from membrane dialysis, centrifuged, and stored at $4^{\circ} \mathrm{C}$.

\section{Enzymatic and hemolytic activity assay of the $V p T L H$.}

Enzymatic activity was measured spectrophotometrically using the lipase/esterase assay described by Nawani et al. in (1998) (Nawani et al. 1998), which was modified to measure the activity of TLH in the presence of egg yolk lecithin as an enzyme activator (Shinoda et al. 1991).

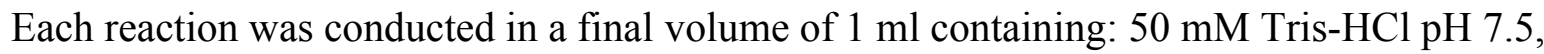
$0.0001 \%$ egg yolk lecithin, $200 \mu \mathrm{M} p$-nitrophenyl laurate (PNPL), and the reaction was started by addition of $10 \mu \mathrm{L}$ of refolded $V p \mathrm{TLH}(6.2 \mu \mathrm{M}$ final concentration). PNPL hydrolysis was measured at a wavelength of $410 \mathrm{~nm}\left(\mathrm{PNPL} \varepsilon_{410 \mathrm{~nm}}=11.8 \mathrm{~cm}-1 \cdot \mu \mathrm{M}-1\right)$ for 5 minutes at a temperature of $37^{\circ} \mathrm{C}$ in a Cary $50 \AA$ UV-VIS spectrophotometer (Varian). Negative control assay consisted of the same reaction without $V p$ TLH. Specific activity was calculated using the equation:

$$
\mathrm{U} / \mathrm{mg} \text { protein }=(\mathrm{m} \cdot \mathrm{V}) /(\varepsilon \cdot \mathrm{p} \cdot \mathrm{l})
$$

where $\mathrm{m}$ is the slope of the reaction; $\mathrm{V}$, the reaction volume; $\mathrm{p}$ the protein concentration $(\mathrm{mg} / \mathrm{ml})$ and 1 , cell path-length in $\mathrm{cm}$.

Hemolytic activity of VpTLH was quantified using Human erythrocytes as a substrate (Malagoli 2007). Erythrocytes were extracted from human blood, which was kindly donated by one male volunteer, who was previously informed about the extraction procedure, the use, and the proper disposal of blood samples, according to the established protocol by the Institutional program of environmental health and safety of the Universidad de Sonora. Also, the volunteer signed an informed consent about her/his participation in this research. Briefly, erythrocytes were isolated from Human blood by centrifugation at $700 \mathrm{rpm}$ for 5 minutes at $4^{\circ} \mathrm{C}$. The plasma was discarded, and erythrocytes were washed three times ( $0.9 \%$ saline solution); they were then carefully resuspended in saline phosphate buffer (PBS; 8 mM Na2HPO4, 2 mM KH2PO4, 37 
$217 \mathrm{mM} \mathrm{NaCl}, 2.7 \mathrm{mM} \mathrm{KCl}, \mathrm{pH}$ 7.4) to 1:200 ratio. Hemolytic activity was measured by the release 218 of hemoglobin at $540 \mathrm{~nm}$ and expressed as a percentage of hemolysis using Tween $20(0.2 \%)$ as 219 a positive control (100\% hemolysis). Erythrocytes autolysis (without enzyme or detergent) was 220 also recorded and subtracted in each assay. Each assay was conducted with $300 \mu \mathrm{L}$ of the 221 erythrocyte suspension, $0.0001 \%$ egg yolk lecithin, and $V p$ TLH ( $0.23 \mathrm{mM}$ final concentration), 222 PBS was added to a final volume of $1 \mathrm{ml}$. Reaction tubes were carefully homogenized and 223 incubated at $37^{\circ} \mathrm{C}$ for $60 \mathrm{~min}$ after tubes were centrifuged ( $2 \mathrm{~min}$ at $700 \mathrm{rpm}$ ), and the 224 supernatant was recovered, and absorbance was recorded at wavelength of $540 \mathrm{~nm}$. All 225 measurements were carried out by triplicate.

226

227

228

229

230

231

232

233

234

235

236

237

238

239

240

241

242

243

244

245

246

247

248

249

250

251

252

253

254

255

256

\section{Biochemical parameters of $V p$ TLH.}

To determine the $\mathrm{pH}$ effect on $\mathrm{VpTLH}$ enzymatic activity, we used several buffer solutions varying $\mathrm{pH}$ values $(5.5,6,7,7.5,8,8.5,9,10$, and 11$)$ under the standard assay mentioned before. PNPL molar extinction coefficient $(\varepsilon)$ was used for each of $\mathrm{pH}$ values: 1.97, 6.52, 11.8, $12.75,13.9,14.3,14.6$ and $14.6\left(\mathrm{~cm}^{-1} \cdot \mu \mathrm{M}^{-1}\right)$, respectively (Kademi et al. 2000). Sodium citrate ( $\mathrm{pH}$ 6), Tris- $\mathrm{HCl}$ for $\mathrm{pH} 7$ to 9, and sodium carbonates ( $\mathrm{pH} 10$ and 11). All buffer's concentration was kept constant at $100 \mathrm{mM}$. The temperature on $V p$ TLH activity was assayed in standard conditions at $\mathrm{pH} 8.0$ by varying temperature assay from $10-80^{\circ} \mathrm{C}$, increasing by $10^{\circ} \mathrm{C}$. Results were expressed as a percentage of residual activity. The temperature with the highest $V p$ TLH activity was used as $100 \%$ of residual activity. Furthermore, $V p$ TLH activation energy (Ea) was calculated by plotting linearized Arrhenius equation:

$\ln (\mathrm{k})=\ln (\mathrm{A})-(\mathrm{Ea} / \mathrm{R}) /(\mathrm{T})$

where slope $\mathrm{m}=-\mathrm{Ea} / \mathrm{R} ; \mathrm{k}$, initial velocities; $\mathrm{T}$, temperature (kelvin) and $\mathrm{R}$, universal gas constant $(\mathrm{J} / \mathrm{molK})$. A negative control without enzyme in each $\mathrm{pH}$ and temperature condition was assayed to discard substrate precipitation or chemical hydrolysis,

For the thermostability test, the enzyme was incubated $15 \mathrm{~min}$ at temperatures from 10 to $80^{\circ} \mathrm{C}$ with $10^{\circ} \mathrm{C}$ intervals. Then enzymatic activity was measured under standard conditions. Residual activity was calculated as a percentage at $V p$ TLH showed the highest activity $(100 \%)$. Additionally, $V p$ TLH melting-temperature $(\mathrm{Tm})$ was obtained by Boltzmann sigmoidal analysis using Prism 5 software (GraphPad®).

\section{Determination of the $V p$ TLH Michaelis-Menten parameters.}

The kinetics parameters $\mathrm{km}$ and $V_{\max }$ of $V p$ TLH were determined from the initial velocities by varying PNPL concentrations from 20 to $400 \mu \mathrm{M}$ using the enzymatic standard conditions mentioned before. Initial velocities were recorded for 2 minutes and were adjusted to the Michaelis-Menten non-linear regression model using the Prism 5 program (GraphPad®). All measurements were carried out by triplicate. Additionally, the Michaelis-Menten constant $\left(K_{m}\right)$, $V_{\max }$, and turnover number $\left(k_{c a t}\right)$ of the enzyme were calculated (Michaelis et al. 2011). 
257

258

259

260

261

262

263

264

265

266

267

268

269

270

271

272

273

274

275

276

277

278

279

280

281

282

283

284

285

286

287

288

289

290

291

292

293

294

295

296

\section{Enzymatic and hemolytic activity inhibition assays}

The following phenolic acids were used: gallic acid (GA), vanillic acid (VA), protocatechuic acid (PR), and chlorogenic (CL). The following flavonoids were used: quercetin, morin, rutin, and epigallocatechin gallate (EGCG) added to the standard enzymatic assay. All flavonoids were used to a final concentration range: $1-20 \mu \mathrm{M}$, while phenolics acids were evaluated at $30 \mu \mathrm{M}$ and $100 \mu \mathrm{M}$. Meanwhile, quercetin, morin, and EGCG were evaluated as an inhibitor for $V p T L H$ hemolytic activity, which was added to the standard hemolytic assay to a final concentration range of 1-20 $\mu \mathrm{M}$. $V p$ TLH residual activity (enzymatic or hemolytic) in the presence of phenolic compounds was calculated as a percentage of $V p$ TLH activity in the absence of phenolic compounds. Flavonoids showed the highest inhibitory effect (see results section), were selected to calculate inhibitor concentration required to reduce $50 \%$ of enzyme activity $\left(\mathrm{IC}_{50}\right)$ using a concentration range of $0.1-50 \mu \mathrm{M}$. The data were normalized using $V p \mathrm{TLH}$ in absence of inhibitor as $100 \%$ of enzymatic activity; then analyzed with the dose-response variable slope model based in Hill Slope equation as described in Prism-5 software (GraphPad®).

\section{Comparison of the TLH sequences from different Vibrio species and homology modeling of} the $V p$ TLH

The amino acid sequence of the $V p \mathrm{TLH}$, with access code Q99289 in the UniProt database, was compared with that of other Vibrio species, including Vibrio cholerae (Q9KMV0), Vibrio alginolyticus (C7EWQ8), Vibrio harveyi (Q2XPT2), Vibrio anguillarum (A0A191WA34) and Vibrio vulnificus (A0A1V8MSL8). These were compared using the ClustalW algorithm (File S1). Based on the alignment obtained, the conserved regions of the sequences were identified, and the presence of the GDSL and SGNH motif, which are specific domains of this type of enzymes. The V $p$ TLH homology model was built using free access algorithms such as PHYRE2, I-TASSER, SWISS-MODEL, and commercial software MOE (File S2). As we expected, all the predicted structures based on 6JL1 crystal structure (TLH from V. vulnificus), showed that the overall structure of predicted models is quite similar in RMSD $=0.324 \AA$. PHYRE2 model (intensive mode) showed the lowest RMSD $(0.223 \AA)$. Therefore this model was used for structural analysis and molecular docking simulations. Structural analysis was performed in UCFS Chimera 1.13.1 (Pettersen et al. 2004) and CCP4-MG programs (McNicholas et al. 2011)

\section{Molecular Docking of the $V p$ TLH substrates and Inhibitors}

Both natural (phosphatidylcholine, PC), synthetic (PNPL) substrate and best IC50 inhibitors were docked into $V p$ TLH active site using AutoDock Vina algorithm in UCFS Chimera 1.13.1 program (McNicholas et al. 2011; Trott \& Olson 2010)(McNicholas et al. 2011; Trott \& Olson 2010). Before docking experiments, both ligands and protein were analyzed in DockPrep function to minimize structure, partial charges calculation, and hydrogen atoms were also added. The 3D structure files of the PNPL, quercetin, morin, and EGCG compounds were obtained from the PubChem database with access codes 74778, 5280343, 5281670, and 65064, respectively. In

Peer) reviewing PDF | (2020:09:52773:1:0:CHECK 6 Nov 2020) 
297

298

299

300

301

302

303

304

305

306

307

308

309

310

311

312

313

314

315

316

317

318

319

320

321

322

323

324

325

326

327

328

329

330

331

332

333

334

335

336

337

338

contrast, PC structural data were obtained from the MOE database. The fpocket2 algorithm predicted a putative ligand pocket cavity in the Phyre2 investigator program (Kelley et al. 2015; Le Guilloux et al. 2009) and the Site Finder function of the MOE program. Sequence alignments located $V p$ TLH active site amino acids and spatial coordinates were established by superposition with $V v$ TLH crystal structure $(\mathrm{RMSD}=0.400 \AA)$. VpTLH final docking area was established in coordinates $\mathrm{X}=24.4143 \AA, \mathrm{Y}=-2.51601 \AA$ and $\mathrm{Z}=-35.3974 \AA$ (volume $=28,802.47 \AA^{3}$ ). For each ligand, 20 poses were generated using Iterated Local search method supported in AutodDocvina (Trott \& Olson 2010); models with low free energy binding force function $(\Delta \mathrm{G})$ were analyzed in Discovery Studio 2019 program (Biova $\left.{ }^{\circledR}\right)$.

\section{Results}

\section{Purification and refolding of $V p$ TLH}

All the over-expression conditions produced the $\operatorname{VpTLH}(\approx 47 \mathrm{kDa})$ protein in the insoluble fraction (Fig. 1A, lanes 1-5). It was in agreement with no phospholipase activity detected on soluble protein fraction by an agar-plates assay using egg yolk lecithin as substrate $(0.1 \%$ egg yolk lecithin, $3 \%$ agar). The higher production of $V p$ TLH was using $0.4 \mathrm{mM}$ IPTG for 16 hours at $25^{\circ} \mathrm{C}$. After that, the inclusion bodies were solubilized in $8 \mathrm{M}$ urea and purified by a single chromatographic step (IMAC) under denaturing conditions. As a result, we obtained a single peak elution at $115 \mathrm{mM}$ imidazole (Fig. 1B), corresponding to a unique SDS-page band of $V p$ TLH molecular weight (Fig. 1A, lanes 6 and 7). The $V p$ TLH was refolded by dialysis, and the esterase activity using PNPL as a substrate on the soluble fraction confirmed that protein was active (Fig. 1C) with a specific activity of $0.47 \mathrm{U} / \mathrm{mg}$ of protein (Shinoda et al. 1991). Figure 1D showed that $V p$ TLH enzymatic activity gradually increased, with the highest PNPL hydrolysis at $10 \mu \mathrm{g}$ lecithin, suggesting that $V p$ TLH could hydrolyze PNPL in lecithin, as early noticed.

Furthermore, the enzymatic activity decreased by $<50 \%$ in the presence of $>50 \mu \mathrm{g}$ lecithin. A quantitative hemolytic activity assay showed that 2-10 $\mu \mathrm{g}$ of purified $V p$ TLH elicits 76-85 \% relative activity (100\% hemolysis was tween-20) (File S3). In this sense, we assayed both enzymatic and hemolytic activity using $10 \mu \mathrm{g}$ of lecithin and confirmed that $V p$ TLH is a lecithindependent protein.

\section{FIGURE 1}

\section{pH and temperature effect on $V p$ TLH activity.}

VpTLH maximum activity was detected at pH 8.0 (100\%), slightly decreasing at $\mathrm{pH} 8.5$ (97\% residual activity). Enzymatic activity suddenly decreased at $\mathrm{pH}<7.5$ and $>9.0$. Contrary, the $V p$ TLH was inactive at acidic medium ( $\mathrm{pH} 6.0)$ while showed low activity $(<20 \%)$ at alkaline pH (Fig. 2A). Amino acid sequence analysis indicated that $V p$ TLH was thermolabile hemolysin (Nishibuchi et al. 1989). Interestingly, to our knowledge, there is no report evaluating the effect of temperature on $\mathrm{VpTLH}$ activity. First, we found that activity increased from $10^{\circ} \mathrm{C}(10 \%$ residual activity) to a maximum level at $50^{\circ} \mathrm{C}$. At higher temperatures $\left(>50^{\circ} \mathrm{C}\right)$, the $V p$ TLH enzymatic activity rapidly decreased, and at $80^{\circ} \mathrm{C}$, no esterase activity was found (Fig. 2B). 
339 These data were analyzed using the linearized Arrhenius equation by plotting natural logarithm

340 (initial velocities) against the inverse of each temperature in Kelvin degrees (Fig. 2C). This

341 analysis showed a single inflection point $\left(50^{\circ} \mathrm{C}\right)$ with linear activity decreasing as a temperature

342 increase (until $80^{\circ} \mathrm{C}$ ), suggesting that temperature above $50^{\circ} \mathrm{C}$ drastically affects $V p T L H$

343 enzymatic activity. The Arrhenius equation calculates the activation energy (Ea), which is the

344 energy that $V p$ TLH requires to hydrolyze PNPL, resulting in $\mathrm{Ea}=26,688 \mathrm{~kJ} / \mathrm{mol}$. Additionally,

345 we evaluated the temperature stability by incubating the enzyme from 10 to $80^{\circ} \mathrm{C}$ for $20 \mathrm{~min}$.

346 After that, the enzyme retained $>80 \%$ residual activity at temperatures below $40^{\circ} \mathrm{C}$. At $60^{\circ} \mathrm{C}$ the

347 enzyme only retained $6 \%$ of residual activity, being inactive at 70 and $80^{\circ} \mathrm{C}$. The data were

348 adjusted to the Boltzmann sigmoid model $\left(\mathrm{R}^{2}=0.9985\right)$, calculating $V p \mathrm{TLH} \mathrm{Tm}=50.94^{\circ} \mathrm{C}($ Fig.

349 2D).

350

351

FIGURE 2

352

353

$V p$ TLH Michalelis-Menten parameters.

354

Initial velocities were measured using PNPL as a substrate from 20 to $400 \mu \mathrm{M}$ (Fig. 3) and

355 showed a typical Michaelis-Menten profile by plotting initial velocities vs. [PNLF]. Data were

356 adjusted to non-linear regression analysis with a correlation factor $\mathrm{R}^{2}=0.9851$. After that, we

357 obtained the $V p$ TLH kinetics parameters, a $V_{\max }=0.7736 \mathrm{U} / \mathrm{mg}( \pm 0.041)$ and a $k_{m}=0.151 \mathrm{mM}$

358 $( \pm 0.017)$ (Fig. 3). Also, the enzyme turnover number $\left(k_{\text {cat }}\right)$ was $37.37 \mathrm{~s}-1$ ( $\left.\pm 1.97 \mathrm{SD}\right)$ (Table 1$)$.

359

360

361

362

363

364

365

366

367

368

369

370

371

372

373

374

375

376

377

378

TLHs Michalelis-Menten kinetic parameters reports are scarce; recently $V v$ TLH kinetics constants were determined using a fluorogenic substrate (Red/Green BODIPY PC-A2) (Wan et al. 2019) and other authors reports PLA2 activity (from snake venom) using 4N3OBA as substrate (Pereanez et al. 2011). In spite of differences in substrates chemical composition used in each report, we observe that $V p$ TLH has the highest Vmax and turn over compared with other TLH and other PLA2 enzymes, lower substrate affinity $\left(k_{\mathrm{m}}\right)$ than $V$. vulinificus TLH (Wan et al. 2019). Table 1 , shows that substrate affinity have variable magnitude among compared enzymes, which is closely-related to substrate differences used (Eisenthal et al. 2007). Meanwhile, Wicka et al 2016, reported similar substrate affinity and $k_{c a t}$ for cold-adapted GDSL-lipase from Pseudomonas sp. S9 using p-nitrophenyl butyrate which has short carbon chain in fatty acids substituent than PNPL (Wicka et al. 2016).

FIGURE 3

TABLE 1

\section{Polyphenols inhibited both $V$ pTLH enzymatic and hemolytic activity}

As shown in fig. 4, phenolic acids GA, PR, and VA inhibited the $V p$ TLH activity by $20 \%$ at 30 $\mathrm{mM}$, while CL does not affect activity compared to control (assay without phenolics acids). Increasing phenolics compounds to $100 \mathrm{mM}$ did not affect the $V p$ TLH activity (p<0.05) (Fig. 4).

Peer) reviewing PDF | (2020:09:52773:1:0:CHECK 6 Nov 2020) 
379 Also, rutin did not affect the activity at all evaluated concentrations; while, quercetin, morin, and 380 EGCG at $20 \mu \mathrm{M}$ (the highest concentration) decreased the activity by $70 \%, 65 \%$, and $67 \%$, 381 respectively (Table 2). At low concentration $(1 \mathrm{mM})$, morin was the most effective to inhibit $382 V p$ TLH (30\% inhibition), whereas, at $10 \mathrm{mM}$, both quercetin and EGCG were also able to reduce 383 activity by $60 \%$. These results suggest that flavonoids were more suitable to inhibit $V p$ TLH 384 phospholipase activity. Additionally, the dose-response analysis showed that quercetin was the 385 best-evaluated inhibitor $\left(\mathrm{IC}_{50}=4.51 \mu \mathrm{M}\right)$. EGCG and morin also exhibited similar $\mathrm{IC}_{50}$ values: $386 \quad 6.290 \mu \mathrm{M}$ and $9.914 \mu \mathrm{M}$, respectively (Fig. 5).

387 The phospholipids are abundant in erythrocytes' membrane cells; thus, VpTLH hemolytic

388

389

390

391

392

393

394

395

396

397

398

399

400

401

402

403

404

405

406

407

408

409

410

411

412

413

414

415

416

417

418

activity in flavonoids at the same concentrations used in enzymatic inhibition experiments (1-20 $\mu \mathrm{M}$ ) was assayed. However, the effect of phenolic compounds on $V p$ TLH hemolytic activity was not evaluated. All flavonoids gradually diminished hemolysis, increasing its concentration, compared to control without flavonoids (Fig. 6). Low concentration (1 and $5 \mu \mathrm{M})$ did not significantly affect erythrocytes lysis, but quercetin and EGCG at 10 and $20 \mu \mathrm{M}$ inhibited the $V p$ TLH hemolytic activity $15 \%$ and $30 \%$, respectively. Morin achieved only $15 \%$ inhibition at the highest evaluated concentration. We could not evaluate flavonoids at concentration $>20$ $\mu \mathrm{M}$ because precipitation of hemolytic assay's components was observed.

FIGURE 4

TABLE 2

FIGURE 5

\section{FIGURE 6}

\section{Homology modeling and docking analysis indicates that $V p$ TLH has conserved folding and} an active site cavity suitable to bind both substrates and inhibitors

We aligned the $V p$ TLH amino acid against TLHs of other pathogenic Vibrio species such as Vibrio alginolyticus $(V a)$, Vibrio harveyi $(V h)$, Vibrio campbelli $(V c)$, Vibrio cholerae $(V c h)$, Vibrio diabolicus $(V d)$, and Vibrio anguillarum (Van). After that, the VpTLH maintained a highsequence identity $>80 \%$ with $V d, V a, V h$, and $V c$; while $V v$, Van, and $V c h$ showed a lower sequence identity being $73 \%, 65 \%$, and $64 \%$, respectively (File S1). This analysis showed that $V p$ TLH has hydrolase/esterase superfamily well-conserved GDSL and SGNH motifs (Akoh et al. 2004; Upton \& Buckley 1995), as was previously reported in other TLHs (Jang et al. 2017; Jia et al. 2010; Wang et al. 2007). Two main domains comprised the TLH sequence, the N-terminal domain included from amino acid residue 24 to 133 (signal peptide 1-23), and C-terminal (also called SGNH domain) comprised 134-418 amino acids (numbering were according to the $V p$ TLH sequence). The N-terminal's biological function is not well defined, while SGNH- 
419 domain is directly related to enzymatic function divided into four blocks that contain invariable 420 catalytic residues (Akoh et al. 2004). GDSL motif is located in block I (139-158) and contained 421 catalytic serine residue (Ser153), while that Gly204, Asn248, and His393 are found in blocks II, 422 III, and V, respectively. SGNH hydrolases have conserved catalytic triad His-Ser-Asp. This last 423 amino acid residue was also found in $V p$ TLH block V (Asp390), and Gly's substitutions were 424 found in Va and Vv TLHs (Jang et al. 2017; Li et al. 2013).

$425 V p$ TLH homology model was built in PHYRE2 using VvTLH crystal structure (PDB: 6JL1) as a 426 template since its share $>74 \%$ sequence identity with $V p$ TLH, and the resulting model showed an

427

428

429

430

431

432

433

434

435

436

437

438

439

440

441

442

443

444

445

446

447

448

449

450

451

452

453

454

455

456

457

458 excellent superposition with the template $(\mathrm{RMSD}=0.256 \AA)$ (Fig. 7A). N-terminal domain (109 amino acid residues) was composed of $\beta$-sheets and three small $\alpha$-helices exposed to solvent while the sizeable C-terminal domain (274 a.a) adopts a typical SGNH $\alpha / \beta / \alpha$ folding related to phospholipase function. Ser-Asp-His catalytic triad and other active site amino acids were located in this domain and distributed in four well-conserved blocks of the SGNH superfamily (Fig. 7A) (Akoh et al. 2004). $\beta$-sheet central core flanked by a-helices compose this domain; all four blocks converge to form ligand pocket cavity as was predicted by the fpocket 2 algorithm in the Phyre2 investigator program (Kelley et al. 2015; Le Guilloux et al. 2009) and Site Finder function of the MOE program. We superposed the VpTLH model to the VvTLH structure, and the catalytic triad was located in the pocket, suggesting it as the $V p$ TLH active site (Fig. 7A and 7B). Although N-terminal domains remain close to the active site, no catalytic function was previously reported for this domain. Nucleophile Ser153 interacted with both Asp390 and His393 that comprised the conserved catalytic triad of serine hydrolases family (Fig. 7C). However, structural and biophysical experimental techniques are necessary to get a more precise model; crystallization assays are in progress in our laboratory with both apo and holo VpTLH. Finally, we used molecular docking simulations of possible interactions with substrates and flavonoids into the C-terminal domain with area $=28.36 \AA$ x $32.65 \AA$ x $31.09 \AA$ following AutoDockVina protocols with centered predicted active site cavity (Fig. 7D). For each ligand, 20 interaction-models were constructed, and we selected the best solution based on the free-energy binding and the position inner the active site. The most favorable interacting-coupling score for substrates were: Phosphatidylcholine $(\mathrm{PC})=-3.9 \mathrm{kcal} / \mathrm{mol}$ and $\mathrm{PNPL}=-4.5 \mathrm{kcal} / \mathrm{mol}$. PC is a natural substrate for TLH and one of the most abundant phospholipids, including phosphatidylserine, in the cellular membrane.

\section{FIGURE 7}

Figure 8 shows the molecular docking and interaction diagram of PC and PNPL into $V p$ TLH active site. PC properly accommodates with the aliphatic chain (glycerol sn-1) buried inside the active site, while fatty acid (glycerol sn-2) was located in the active site surface and polar substituent (choline) is more exposed to the solvent. PC interacted with several active site amino acids by hydrogen bonds between Gln292 and OH-groups (both fatty acids) and Asn254 with 
459 phosphate group; also, Lys303 stabilize phosphate group by a saline bridge. Asn252, Ala206, 460 and Tyr253 stabilize fatty acids chains and choline methyl groups by aliphatic C-H interactions. 461 Non-canonical substrate interaction prediction showed an embedded PNPL into VpTLH active 462 site. The $p$-nitrophenol ring shows hydrophobic $\pi$-alkyl- interaction to Ala206 (coordination), 463 and the carboxylate was stabilized by hydrogen bonds with Tyr360 lateral chain OH-. 14-C fatty 464 acid chain (laurate) was located in a similar arrangement as the second PC fatty acid substituent 465 (sn-2). Interestingly, catalytic residues (SGNH) did not interact with both substrates in docking 466 experiments under the used conditions. Similar results were also reported in the crystallographic

467 468 469

470

471

472

473

474

475

476

477

478

479

480

481

482

483

484

485

486

487

488

489

490

491

492

493

494

495

496

497

498

model of $V v$ TLH single mutant (Gly389Asn) in complex with hexamethylene glycol, concluding that TLHs could elicit conformational flexibility upon substrate binding (Wan et al. 2019)

\section{FIGURE 8}

Flavonoids that inhibited both enzymatic and hemolytic activity (quercetin, morin, and EGCG) were also docked into the predicted $V p$ TLH active site (Fig. 9A). Interactions diagram showed that free energy binding was favorable to EGCG>morin $>$ quercetin with scores: $-7.9,-7.2$, and $6.4 \mathrm{kcal} / \mathrm{mol}$, respectively. EGCG (gallic acid substituent) and quercetin (ring A) were buried into the active site as observed with both substrates. Ala206 $\pi$-alkyl stabilizes EGCG gallic acid and quercetin ring A, while hydrogen bridge Gln292 with quercetin 1' -oxygen and EGCG 4'hydroxyl group (Fig. 9B). Such interactions were not observed in morin docking simulations, positioned near active site surface throw $\pi$-anion interaction with Glu300 (ring B and C) and hydrogen bridge to Thr297 and Van der Waals forces with oxygen groups located in morin ring B (Fig. 9B). Morin and quercetin have identical chemical formula and molar mass but differ in hydroxyl; morin is 2', 4' while quercetin is 3', 4' orientation in ring B; such could be related to differences in $\Delta G$ scores and active site interactions (Xiao et al. 2012). EGCG and quercetin displayed similar disposition into active site $V p$ TLH, as observed with substrates evaluated. These results are consistent with the inhibition experiment; therefore, both flavonoids could be suitable compounds for chemical modification for structure/function studies and evaluate inhibition/attenuation capacity during $V$. parahaemolyticus infection.

\section{FIGURE 9}

\section{Discussion}

We performed several strategies to obtain soluble $V p T L H$, such as testing several cultures and overexpression conditions and using a protein with and without a signal peptide. Unfortunately, in all strategies, the $V p$ TLH was obtained as inclusion bodies. The VpTLH was refolded into active form eliminating chaotropic agents by dialysis, recovering $15 \mathrm{mg}$ of purified active enzyme per liter of culture media. Contrary, several studies have been reported with different results. Shinoda et al. (1991) first reported the recombinant production of $V p$ TLH as an active soluble protein from the periplasm of E. coli(Shinoda et al. 1991). Recombinant hemolysins 
499 TDH, TRH, and TLH from $V p$ also were expressed as inactive form and renatured by carbamide 500 gradient dialysis (Zhao et al. 2011). Despite these differences, recombinant VpTLH showed 501 lecithin-dependent phospholipase and hemolytic activity as other Vibrio TLHs (Jia et al. 2010; Li 502 et al. 2013; Zhao et al. 2011). GDSL-esterases and SGNH-hydrolases enzymes have flexible 503 active site exhibiting conformational changes upon substrate binding and favoring enzyme 504 catalysis (Akoh et al. 2004; Wan et al. 2019). Lecithin could induce VpTLH local or global 505 conformational changes in active site vicinity, allowing hydrolyzing PNPL, which will require 506 further studies using biophysical and biochemical approaches to demonstrate this hypothesis. 507 Lecithin-dependent hemolysins are widely overexpressed between the Vibrionaceae family's 508 microorganisms, and they showed different temperature sensitivities. For example, $V$. 509 anguillarum hemolysin has a broad optimal temperature from 37 to $64^{\circ} \mathrm{C}(\mathrm{Li}$ et al. 2013), while 510 the optimal temperature in $V$. harveyi hemolysin was $37^{\circ} \mathrm{C}$ and it was inactivated by $30 \mathrm{~min}$ at $51165^{\circ} \mathrm{C}$ (Zhong et al. 2006). Miwatani et al. (1972) first describe that $V p$ strains secreted hemolytic 512 factors showing different behavior with the temperature increase from $60^{\circ} \mathrm{C}$ (partially 513 inactivated) to $90^{\circ} \mathrm{C}$ (entirely inactive) (Miwatani et al. 1972; Takeda et al. 1974). Later, 514 Taniguchi et al. (1985) identified another hemolysin that was wholly inactivated by $10 \mathrm{~min}$ at $51560^{\circ} \mathrm{C}$ (Taniguchi et al. 1986).

516 Our results suggest that $V p$ TLH maximum enzymatic activity was at $50^{\circ} \mathrm{C}$ and suddenly 517 decreases to entirely inactive at $80^{\circ} \mathrm{C}$, while gradually decreasing activity at low temperatures $518\left(10-40^{\circ} \mathrm{C}\right.$ ) retaining $80 \%$ residual activity at $37^{\circ} \mathrm{C}$. The linearized Arrhenius equation (Fig. $2 \mathrm{C}$ ) 519 suggested that $\mathrm{V} p \mathrm{TLH}$ follows a one-steady denaturation process without apparently 520 intermediate transition states with $\mathrm{Ea}=26.6 \mathrm{kcal} / \mathrm{mol}$ (Segel 1975). This behavior also was 521 described in psychrophilic enzymes; that show high structural flexibility to diminish activation 522 energy during catalysis (Feller \& Gerday 1997). Furthermore, $V p$ TLH lost $50 \%$ of enzymatic 523 activity by $30 \mathrm{~min}$ at $50.9^{\circ} \mathrm{C}$ and was inactivated at $70^{\circ} \mathrm{C} . V p \mathrm{TLH}$ melting temperature is related 524 to linearized Arrhenius plot suggesting that temperatures $>50^{\circ} \mathrm{C}$ induce the loss of enzymatic 525 activity by local (active site) or global structural destabilization. Finally, these results indicate 526 that $V p$ TLH is a thermolabile enzyme; however, other thermodynamic and structural approaches 527 are necessary to understand the TLH inactivation process.

528 TLH is a ubiquitous protein among Vibrionaceae species (Wang et al. 2007), and it is a 529 molecular marker to both clinical and environmental $V$. parahaemolyticus strains (Bej et al. 530 1999; Chen et al. 2017). VpTLH can lysate both human and fish erythrocytes through 531 phospholipase A2 activity and showed cytotoxicity activity against human cells (Wang et al. 532 2012; Wang et al. 2015). In this sense, we found that flavonoids were more effective than 533 phenolic acid to inhibit $V p$ TLH enzymatic activity and hemolytic capacity against human 534 erythrocytes (Table 2 and Fig. 5). TLHs inhibition studies are scarce, but recently, resveratrol at $5358 \mu \mathrm{g} / \mathrm{ml}$ inhibits almost $100 \% \mathrm{VhTLH}$ hemolytic activity by binding into the active site and at 2 $536 \mu \mathrm{g} / \mathrm{ml}$ reduced cell damage caused by $V h T L H$ (Zhao et al. 2020). Resveratrol is a polyphenol 537 belonging to stilbenes with a characteristic nucleus of 1,2-diphenylethylene that could have 538 hydroxyl substitutions in aromatics rings, as occurring in flavonoids and other phenolics 
539 compounds, and therefore share biological and physicochemical activities with those (Han et al. 540 2007). Other studies are focused on antibody neutralization using phage display technologies 541 (Wang et al. 2012). Polyphenols have inhibitory activity against PLA2 from snake venoms;

542 Crotalus durissus terrificus PLA2 enzymatic activity was inhibited (40\%) with $50 \mu \mathrm{M}$. Also, 543 Iglesias et al. (2005) isolated PLA2 from tropical rattlesnake (C. durissus sub cascavella) and 544 found that morin $20 \mathrm{uM}$ reduce PLA2 activity by $70 \%$. EGCG showed the best inhibitory activity

545

546

547

548

549

550

551

552

553

554

555

556

557

558

559

560

561

562

563

564

565

566

567

568

569

570

571

572

573

574

575

576

577

578

against $C$. durissus sub. Cumanensis PLA2 compared to phenolics compounds as cafeic and ferulic acid. All evaluated compounds belong to the flavonol group (3-hydroxy flavone) that share a base structure with various biological properties depending on hydroxyl substitutions and different conjugations (Massi et al. 2017). These observations are consistent with our results because quercetin or quercetin-derivatives, such as glycosides and gallic acid or hydroxyl substitutions, inhibited the $V p$ TLH. Quercetin $\left(\mathrm{IC}_{50}=4.5 \mu \mathrm{M}\right)$ was a 2 -fold higher $V p \mathrm{TLH}$ inhibitor than morin $\left(\mathrm{IC}_{50}=9.9 \mu \mathrm{M}\right)$, which has a single change in one hydroxyl position in $3^{\prime}$ and 2 ', respectively. While EGCG contains a 3'-gallic acid conjugation and an additional 5'-hydroxyl group, these substitutions could be associated with the slight decrease in inhibitory effect than quercetin, but it was more effective than morin. Quercetin 3-O-rhamnosylglucoside conjugation (rutin) may affect binding to $\mathrm{VpTLH}$ active site and thus could not inhibit the enzyme. Changes in hydroxyl group positions ( $3^{\prime}, 4^{\prime}$ and $\left.5^{\prime}\right)$ could be affecting the $V p$ TLH inhibitory capacity. TLHs show high conserved amino acid sequence among Vibrios species ( $>70 \%)$ and maintains the characteristic catalytic triad (Ser-His-Asp) except for $V v$ and Van in which acidic residue was substituted by chloride atom during catalysis (Wan et al. 2019). We obtained a successful model of $V p \mathrm{TLH}$ using a recently solved $V v \mathrm{TLH}$ crystal structure as a template (PDB: 6JL1). VpTLH has a C-terminal domain with typical GDSL a/b hydrolase folding, located at the active site (Akoh et al. 2004; Wan et al. 2019). Molecular docking experiments suggested that quercetin, EGCG, and morin could interact with $V p$ TLH active site and PC and PNPL with free energy binding values from -3.9 and $-7.9 \mathrm{kcal} / \mathrm{mol}$. Zhao et al. (2020) reported similar results by docking resveratrol to $V h \mathrm{TLH}$ and found that binding energy was $-6.0 \mathrm{kcal} / \mathrm{mol}$. Resveratrol binds to VhTLH active site through Lue247 and Tyr368 by $\pi$-alkyl interactions and hydrogen bonds, respectively (Zhao et al. 2020). Also, both residues are essential during the resveratrol binding process to $V h T L H$ and are straightly related to hemolytic activity inhibition. VpTLH interaction diagrams showed that Ala206 and Gln292 could be important for binding both substrate (PC) and inhibitors (quercetin and EGCG) to the active site by hydrogen bonds with Gln142 and $\pi$-alkyl interactions with Ala206. Thus, we suggest that both residues could be critical during the binding process to $V p T L H$. Although the study of such interactions could be the target of future studies using conjugated phenolics compounds that may enhance $V p$ TLH inhibition, future biochemical and structure-function studies should examine these hypotheses. In summary, our results showed that $V p$ TLH has conserved GDSL hydrolase folding with conserved active sites composed by catalytic triad Ser-His-Asp. Biochemical studies demonstrated that polyphenols as quercetin, EGCG, and morin were suitable $V p$ TLH inhibitors, and molecular docking suggests the interaction with the active site. Future research should also

Peer) reviewing PDF | (2020:09:52773:1:0:CHECK 6 Nov 2020) 
579 focus on evaluating antibacterial or/and bacteriostatic effects of flavonoids on $V$.

580 parahaemolyticus and bacterial infection in a host such as shrimp or fish.

581

582 Conclusions

583 In the present study, we purified in two steps from inclusion bodies a functional $\mathrm{VPTLH}$. The

584 enzyme showed thermolabile characteristics compared to TDH and TRH. Furthermore, the

585 kinetic parameter $k_{\mathrm{m}}$ was similar to that described for other GDSL enzymes. On the other hand,

586 quercetin, EGCG, and morin inhibited $V p T L H$ activity possibly due to the active site binding, as

587 predicted by molecular docking and showed similar structural orientation into active site $V p$ TLH

588 compared to PC and PNPL. Therefore, the flavonoids that we evaluated and others with similar

589 physicochemical properties could be suitable compounds to chemical modification for

590 structure/function studies and to evaluate the inhibition/attenuation capacity during $V$.

591 parahaemolyticus infection.

592

593

\section{Acknowledgements}

594

We thank Cesar Otero-León for technical support.

595

596

597

598

599

600

601

602

603

604

605

606

607

608

609

610

611

612

613

614

615

616

617

618

619

Akoh CC, Lee GC, Liaw YC, Huang TH, and Shaw JF. 2004. GDSL family of serine esterases/lipases. Prog Lipid Res 43:534-552. 10.1016/j.plipres.2004.09.002

Bej AK, Patterson DP, Brasher CW, Vickery MC, Jones DD, and Kaysner CA. 1999. Detection of total and hemolysin-producing Vibrio parahaemolyticus in shellfish using multiplex PCR amplification of tl, tdh and trh. J Microbiol Methods 36:215-225. 10.1016/s01677012(99)00037-8

Carter P, and Wells JA. 1988. Dissecting the catalytic triad of a serine protease. Nature 332:564568.

Chen AJ, Hasan NA, Haley BJ, Taviani E, Tarnowski M, Brohawn K, Johnson CN, Colwell RR, and Huq A. 2017. Characterization of Pathogenic Vibrio parahaemolyticus from the Chesapeake Bay, Maryland. Front Microbiol 8:2460. 10.3389/fmicb.2017.02460

Cheynier V. 2012. Phenolic compounds: from plants to foods. Phytochemistry Reviews 11:153177. 10.1007/s11101-012-9242-8

Cotrim CA, de Oliveira SCB, Diz Filho EBS, Fonseca FV, Baldissera L, Antunes E, Ximenes RM, Monteiro HSA, Rabello MM, Hernandes MZ, de Oliveira Toyama D, and Toyama MH. 2011. Quercetin as an inhibitor of snake venom secretory phospholipase A2. Chemico-Biological Interactions 189:9-16. https://doi.org/10.1016/j.cbi.2010.10.016

Cuéllar-Anjel J. 2012. Síndrome de mortalidad temprana (EMS). Institute for International Cooperation in Animal Biologics. 
620 Dy RL, Rigano LA, and Fineran PC. 2018. Phage-based biocontrol strategies and their

621

622

623

624

625

626

627

628

629

630

631

632

633

634

635

636

637

638

639

640

641

642

643

644

645

646

647

648

649

650

651

652

653

654

655

656

657

658

659

660

661

662

663

664

665

666 application in agriculture and aquaculture. Biochem Soc Trans 46:1605-1613. 10.1042/BST20180178

Eisenthal R, Danson MJ, and Hough DW. 2007. Catalytic efficiency and kcat/KM: a useful comparator? Trends in Biotechnology 25:247-249. https://doi.org/10.1016/j.tibtech.2007.03.010

Feller G, and Gerday C. 1997. Psychrophilic enzymes: molecular basis of cold adaptation. Cellular and Molecular Life Sciences CMLS 53:830-841. 10.1007/s000180050103

Flores-Diaz M, Monturiol-Gross L, Naylor C, Alape-Giron A, and Flieger A. 2016. Bacterial Sphingomyelinases and Phospholipases as Virulence Factors. Microbiol Mol Biol Rev 80:597-628. 10.1128/MMBR.00082-15

Freire-Moran L, Aronsson B, Manz C, Gyssens IC, So AD, Monnet DL, Cars O, and Group EEW. 2011. Critical shortage of new antibiotics in development against multidrug-resistant bacteria-Time to react is now. Drug Resist Updat 14:118-124. 10.1016/j.drup.2011.02.003

Han X, Shen T, and Lou H. 2007. Dietary Polyphenols and Their Biological Significance. International Journal of Molecular Sciences 8:950-988.

Iglesias CV, Aparicio R, Rodrigues-Simioni L, Camargo EA, Antunes E, Marangoni S, de Oliveira Toyama D, Beriam LO, Monteiro HS, and Toyama MH. 2005. Effects of morin on snake venom phospholipase A2 (PLA2). Toxicon 46:751-758. 10.1016/j.toxicon.2005.07.017

Jang KK, Lee ZW, Kim B, Jung YH, Han HJ, Kim MH, Kim BS, and Choi SH. 2017. Identification and characterization of Vibrio vulnificus plpA encoding a phospholipase A2 essential for pathogenesis. J Biol Chem 292:17129-17143. 10.1074/jbc.M117.791657

Jia A, Woo NY, and Zhang XH. 2010. Expression, purification, and characterization of thermolabile hemolysin (TLH) from Vibrio alginolyticus. Dis Aquat Organ 90:121-127. 10.3354/dao02225

Kademi A, Ait-Abdelkader N, Fakhreddine L, and Baratti JC. 2000. Characterization of a new thermostable esterase from the moderate thermophilic bacterium Bacillus circulans. Journal of Molecular Catalysis B: Enzymatic 10:395-401. https://doi.org/10.1016/S13811177(99)00111-3

Kelley LA, Mezulis S, Yates CM, Wass MN, and Sternberg MJ. 2015. The Phyre2 web portal for protein modeling, prediction and analysis. Nat Protoc 10:845-858. 10.1038/nprot.2015.053

Klein SL, Gutierrez West CK, Mejia DM, and Lovell CR. 2014. Genes similar to the Vibrio parahaemolyticus virulence-related genes tdh, th, and vscC2 occur in other vibrionaceae species isolated from a pristine estuary. Appl Environ Microbiol 80:595-602. 10.1128/AEM.02895-13

Laemmli UK. 1970. Cleavage of structural proteins during the assembly of the head of bacteriophage T4. Nature 227:680-685. 10.1038/227680a0

Le Guilloux V, Schmidtke P, and Tuffery P. 2009. Fpocket: an open source platform for ligand pocket detection. BMC Bioinformatics 10:168. 10.1186/1471-2105-10-168

Li L, Meng H, Gu D, Li Y, and Jia M. 2019. Molecular mechanisms of Vibrio parahaemolyticus pathogenesis. Microbiol Res 222:43-51. 10.1016/j.micres.2019.03.003

Li L, Mou X, and Nelson DR. 2013. Characterization of Plp, a phosphatidylcholine-specific phospholipase and hemolysin of Vibrio anguillarum. BMC Microbiol 13:271. 10.1186/1471-2180-13-271

Peer) reviewing PDF | (2020:09:52773:1:0:CHECK 6 Nov 2020) 
667

668

669

670

671

672

673

674

675

676

677

678

679

680

681

682

683

684

685

686

687

688

689

690

691

692

693

694

695

696

697

698

699

700

701

702

703

704

705

706

707

708

709

710

711

Lindahl M, and Tagesson C. 1997. Flavonoids as Phospholipase A2 Inhibitors: Importance of Their Structure for Selective Inhibition of Group II Phospholipase A2. Inflammation 21:347-356. 10.1023/A:1027306118026

Malagoli D. 2007. A full-length protocol to test hemolytic activity of palytoxin on human erythrocytes. Invertebrate Survival Journal 4:92-94.

Massi A, Bortolini O, Ragno D, Bernardi T, Sacchetti G, Tacchini M, and De Risi C. 2017. Research Progress in the Modification of Quercetin Leading to Anticancer Agents. Molecules 22. 10.3390/molecules22081270

McNicholas S, Potterton E, Wilson KS, and Noble ME. 2011. Presenting your structures: the CCP4mg molecular-graphics software. Acta Crystallogr D Biol Crystallogr 67:386-394. $10.1107 / \mathrm{S} 0907444911007281$

Michaelis L, Menten ML, Johnson KA, and Goody RS. 2011. The original Michaelis constant: translation of the 1913 Michaelis-Menten paper. Biochemistry 50:8264-8269. $10.1021 /$ bi201284u

Miwatani T, Takeda Y, Sakurai J, Yoshihara A, and Taga S. 1972. Effect of Heat (Arrhenius Effect) on Crude Hemolysin of \&lt;em\&gt;Vibrio parahaemolyticus\&lt;/em\&gt. Infection and Immunity 6:1031.

Murakami M, and Kudo I. 2002. Phospholipase A2. J Biochem 131:285-292. 10.1093/oxfordjournals.jbchem.a003101

Nawani N, Dosanjh NS, and Kaur J. 1998. A novel thermostable lipase from a thermophilic Bacillus sp.: characterization and esterification studies. Biotechnology Letters 20:9971000.

Nishibuchi M, Taniguchi T, Misawa T, Khaeomanee-Iam V, Honda T, and Miwatani T. 1989. Cloning and nucleotide sequence of the gene (trh) encoding the hemolysin related to the thermostable direct hemolysin of Vibrio parahaemolyticus. Infect Immun 57:2691-2697.

Özçelik B, Kartal M, and Orhan I. 2011. Cytotoxicity, antiviral and antimicrobial activities of alkaloids, flavonoids, and phenolic acids. Pharmaceutical Biology 49:396-402. $10.3109 / 13880209.2010 .519390$

Panche AN, Diwan AD, and Chandra SR. 2016. Flavonoids: an overview. Journal of Nutritional Science 5:e47. 10.1017/jns.2016.41

Pereanez JA, Nunez V, Patino AC, Londono M, and Quintana JC. 2011. Inhibitory effects of plant phenolic compounds on enzymatic and cytotoxic activities induced by a snake venom phospholipase A2. Vitae 18:295-304.

Perez-Acosta JA, Martinez-Porchas M, Elizalde-Contreras JM, Leyva JM, Ruiz-May E, GollasGalvan T, Martinez-Cordova LR, and Huerta-Ocampo JA. 2018. Proteomic profiling of integral membrane proteins associated to pathogenicity in Vibrio parahaemolyticus strains. Microbiol Immunol 62:14-23. 10.1111/1348-0421.12556

Petrescu AM, Paunescu V, and Ilia G. 2019. The antiviral activity and cytotoxicity of 15 natural phenolic compounds with previously demonstrated antifungal activity. Journal of Environmental Science and Health, Part B 54:498-504. 10.1080/03601234.2019.1574176

Pettersen EF, Goddard TD, Huang CC, Couch GS, Greenblatt DM, Meng EC, and Ferrin TE. 2004. UCSF Chimera--a visualization system for exploratory research and analysis. $J$ Comput Chem 25:1605-1612. 10.1002/jcc.20084

Raghunath P. 2014. Roles of thermostable direct hemolysin (TDH) and TDH-related hemolysin (TRH) in Vibrio parahaemolyticus. Front Microbiol 5:805. 10.3389/fmicb.2014.00805

Peer) reviewing PDF | (2020:09:52773:1:0:CHECK 6 Nov 2020) 
712

713

714

715

716

717

718

719

720

721

722

723

724

725

726

727

728

729

730

731

732

733

734

735

736

737

738

739

740

741

742

743

744

745

746

747

748

749

750

751

752

753

754

755

756
Saito S, Iwade Y, Tokuoka E, Nishio T, Otomo Y, Araki E, Konuma H, Nakagawa H, Tanaka H, Sugiyama K, Hasegawa A, Sugita-Konishi Y, and Hara-Kudo Y. 2015. Epidemiological evidence of lesser role of thermostable direct hemolysin (TDH)-related hemolysin (TRH) than TDH on Vibrio parahaemolyticus pathogenicity. Foodborne Pathog Dis 12:131-138. 10.1089/fpd.2014.1810

Santos L, and Ramos F. 2018. Antimicrobial resistance in aquaculture: Current knowledge and alternatives to tackle the problem. Int J Antimicrob Agents 52:135-143. 10.1016/j.ijantimicag.2018.03.010

Segel IH. 1975. Enzyme kinetics: behavior and analysis of rapid equilibrium and steady state enzyme systems: Wiley.

Shinoda S, Matsuoka H, Tsuchie T, Miyoshi S, Yamamoto S, Taniguchi H, and Mizuguchi Y. 1991. Purification and characterization of a lecithin-dependent haemolysin from Escherichia coli transformed by a Vibrio parahaemolyticus gene. J Gen Microbiol 137:2705-2711. 10.1099/00221287-137-12-2705

Takeda Y, Hori Y, and Miwatani T. 1974. Demonstration of a temperature-dependent inactivating factor of the thermostable direct hemolysin in Vibrio parahaemolyticus. Infection and Immunity 10:6-10.

Taniguchi H, Hirano H, Kubomura S, Higashi K, and Mizuguchi Y. 1986. Comparison of the nucleotide sequences of the genes for the thermostable direct hemolysin and the thermolabile hemolysin from Vibrio parahaemolyticus. Microb Pathog 1:425-432. 10.1016/0882-4010(86)90004-5

Trott O, and Olson AJ. 2010. AutoDock Vina: improving the speed and accuracy of docking with a new scoring function, efficient optimization, and multithreading. J Comput Chem 31:455-461. 10.1002/jcc.21334

Upton C, and Buckley JT. 1995. A new family of lipolytic enzymes? Trends Biochem Sci 20:178-179. 10.1016/s0968-0004(00)89002-7

Wan Y, Liu C, and Ma Q. 2019. Structural analysis of a Vibrio phospholipase reveals an unusual Ser-His-chloride catalytic triad. J Biol Chem 294:11391-11401. 10.1074/jbc.RA119.008280

Wang R, Fang S, Wu D, Lian J, Fan J, Zhang Y, Wang S, and Lin W. 2012. Screening for a single-chain variable-fragment antibody that can effectively neutralize the cytotoxicity of the Vibrio parahaemolyticus thermolabile hemolysin. Appl Environ Microbiol 78:49674975. 10.1128/AEM.00435-12

Wang R, Zhong Y, Gu X, Yuan J, Saeed AF, and Wang S. 2015. The pathogenesis, detection, and prevention of Vibrio parahaemolyticus. Front Microbiol 6:144.

10.3389/fmicb.2015.00144

Wang SX, Zhang XH, Zhong YB, Sun BG, and Chen JX. 2007. Genes encoding the Vibrio harveyi haemolysin (VHH)/thermolabile haemolysin (TLH) are widespread in vibrios. Wei Sheng Wu Xue Bao 47:874-881.

Wicka M, Wanarska M, Krajewska E, Pawlak-Szukalska A, Kur J, and Cieslinski H. 2016. Cloning, expression, and biochemical characterization of a cold-active GDSL-esterase of a Pseudomonas sp. S9 isolated from Spitsbergen island soil. Acta Biochim Pol 63:117125. 10.18388/abp.2015_1074

Xiao ZP, Wang XD, Peng ZY, Huang S, Yang P, Li QS, Zhou LH, Hu XJ, Wu LJ, Zhou Y, and Zhu HL. 2012. Molecular docking, kinetics study, and structure-activity relationship

Peer] reviewing PDF | (2020:09:52773:1:0:CHECK 6 Nov 2020) 
757

758

759

760

761

762

763

764

765

766

767

768

769

770

771

772

773

774 analysis of quercetin and its analogous as Helicobacter pylori urease inhibitors. $J$ Agric Food Chem 60:10572-10577. 10.1021/jf303393n

Yanagase Y, Inoue K, Ozaki M, Ochi T, and Amano T. 1970. Hemolysins and related enzymes of Vibrio parahaemolyticus. I. Identification and partial purification of enzymes. Biken $J$ 13:77-92.

Zeng S, Hou D, Liu J, Ji P, Weng S, He J, and Huang Z. 2019. Antibiotic supplement in feed can perturb the intestinal microbial composition and function in Pacific white shrimp. Appl Microbiol Biotechnol 103:3111-3122. 10.1007/s00253-019-09671-9

Zhao X, Guo Y, Ni P, Liu J, Wang F, Xing Z, and Ye S. 2020. Resveratrol inhibits the virulence of Vibrio harveyi by reducing the activity of Vibrio harveyi hemolysin. Aquaculture $522: 735086$.

Zhao Y, Tang X, and Zhan W. 2011. Cloning, expressing, and hemolysis of tdh, trh and th genes of Vibrio parahaemolyticus. Journal of Ocean University of China 10:275. 10.1007/s11802-011-1801-x

Zhong Y, Zhang XH, Chen J, Chi Z, Sun B, Li Y, and Austin B. 2006. Overexpression, purification, characterization, and pathogenicity of Vibrio harveyi hemolysin VHH. Infect Immun 74:6001-6005. 10.1128/IAI.00512-06 


\section{Table $\mathbf{1}$ (on next page)}

Michaelis-Menten kinetic parameters of TLH and other enzymes with similar catalytic properties, including GDLS-lipases and snake venom PLA ${ }_{2}$

NR: not reported.EstS9 Psp., GDSL-lipase Pseudomonas sp Cdc, C. durissus sub cascavella Cdt, Crotalus durissus terrificus 
1 Table I. Michaelis-Menten kinetic parameters of TLH and other enzymes with similar 2 catalytic properties, including GDLS-lipases and snake venom PLA 2 .

\begin{tabular}{|c|c|c|c|c|}
\hline $\begin{array}{c}\text { Enzyme and } \\
\text { source }\end{array}$ & $k_{m}(\mathbf{m M})$ & $V_{\max }(\mathrm{U} / \mathrm{mg})$ & $k_{c a t}\left(\mathrm{~s}^{-1}\right)$ & Reference \\
\hline TLH $V p$ & 0.151 & 0.7736 & 37.37 & This work \\
\hline TLH WT $V v$ & 0.020 & 0.0216 & 0.051 & Ye et al., 2019 \\
\hline TLH G389D $V v$ & 0.0209 & 0.0118 & 0.028 & Ye et al., 2019 \\
\hline $\mathrm{sPLA}_{2} C d c$ & 60 & 0.0034 & NR & 2009 \\
\hline $\mathrm{sPLA}_{2} C d t$ & 31 & 0.0082 & NR & Oliveira et al., 2002 \\
\hline EstS9N Psp. & 0.161 & NR & 3.31 & Wicka et al., 2016 \\
\hline
\end{tabular}

NR: not reported.

$4 \quad$ EstS9 Psp., GDSL-lipase Pseudomonas $s p$

$5 \quad C d c, C$. durissus sub cascavella

$6 \quad C d t$, Crotalus durissus terrificus 


\section{Table 2 (on next page)}

Inhibition percentage of VpTLH enzymatic activity by flavonoids.

atistically significant values $(p<0.05)$ are represented with an $* .{ }^{b}$ Epigallocatechingallate ${ }^{c} \mathrm{NI}$ : not inhibit. 
1

Table II. Inhibition percentage of $V p$ TLH enzymatic activity by flavonoids.

\begin{tabular}{cccccc}
\hline $\begin{array}{c}\text { Compound/Dose } \\
(\boldsymbol{\mu M})\end{array}$ & $\mathbf{1}$ & $\mathbf{5}$ & $\mathbf{1 0}$ & $\mathbf{2 0}$ & $\boldsymbol{p}$ value $^{\mathbf{a}}$ \\
\hline Quercetin & $14.9 \pm 4.2$ & $34.5 \pm 4.0$ & $62.2 \pm 7.4$ & $70.3 \pm 8.5$ & $0.011^{*}$ \\
Morin & $30.3 \pm 2.1$ & $38.7 \pm 8.3$ & $45.4 \pm 6.2$ & $65.5 \pm 5.4$ & $0.018^{*}$ \\
EGCG $^{\mathrm{b}}$ & $16.8 \pm 7.2$ & $44.7 \pm 6.8$ & $60.3 \pm 13.0$ & $67.8 \pm 7.2$ & $0.020^{*}$ \\
Rutin & $\mathrm{NI}^{\mathrm{C}}$ & $\mathrm{NI}$ & $\mathrm{NI}$ & $\mathrm{NI}$ & ----- \\
\hline
\end{tabular}

${ }^{a}$ Statistically significant values $(p<0.05)$ are represented with an *.

${ }^{\mathrm{b}}$ Epigallocatechingallate

${ }^{c}$ NI: not inhibit. 
Figure 1

Recombinant overexpression, purification, and refolding of VpTLH.

(A) 12\% SDS-PAGE of the recombinant over-expression process and in vitro refolding of purified VpTLH. M: molecular weight marker; lane 1 and 2: the soluble and insoluble fractions of non-induced E. coli culture. Lane 3 and 4: insoluble and soluble fraction 16 hours after the addition of IPTG to the culture; lane 5, solubilized inclusion bodies (8M urea); lane 6, purified VPTLH under denaturing conditions; lane 7, in vitro refolded VpTLH. (B) Chromatogram of VpTLH IMAC purification under denaturing conditions. (C) Esterase activity assay of refolded VpTLH. $410 \mathrm{~nm}$ absorbance increases as PNPL hydrolysis releasing p-nitrophenol. The assay was performed by triplicate; PNPL self-hydrolysis (control) was assayed without VpTLH. (D) Effect of lecithin on VpTLH enzymatic activity.

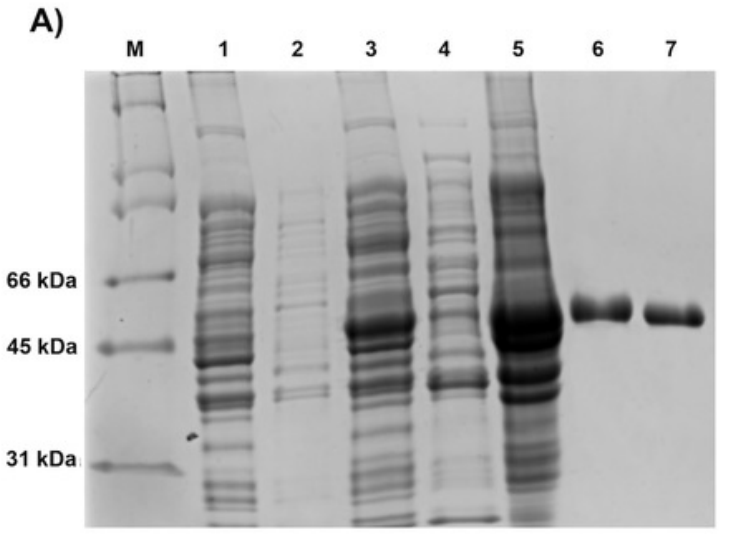

C)

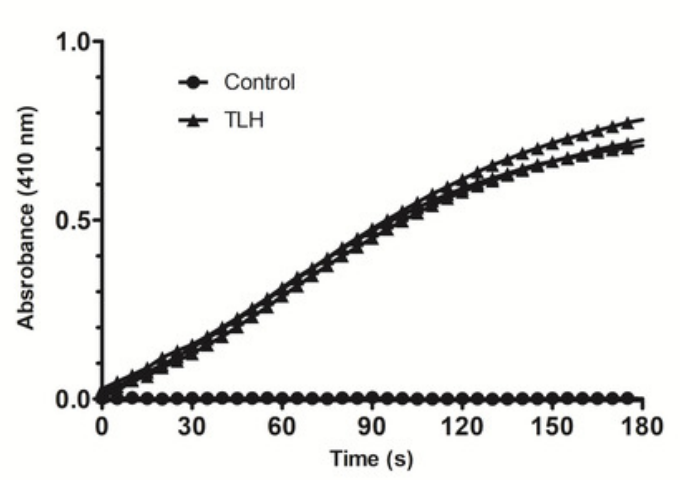

\section{B)}

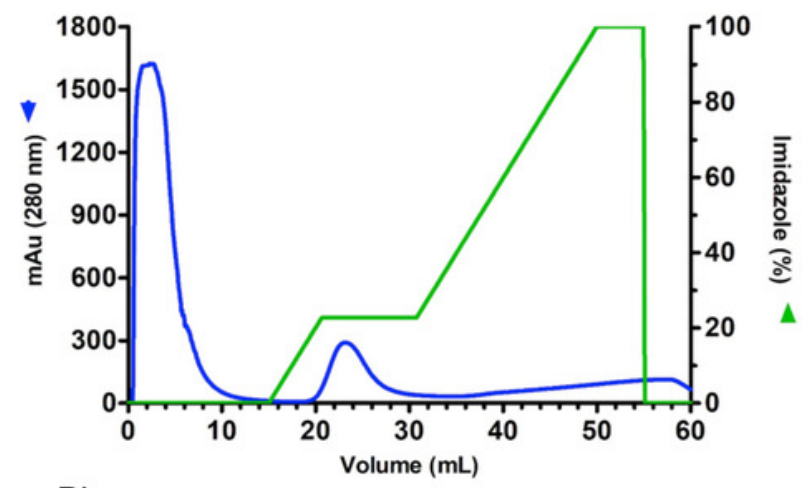

D)

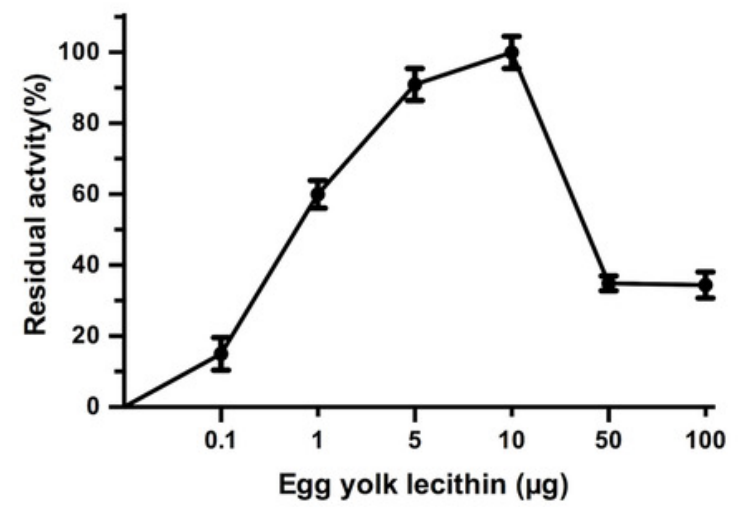




\section{Figure 2}

Biochemical properties of $\mathrm{VPTLH}$

Enzymatic activity was calculated as the residual activity respect to the highest value detected in each assay. Results were the mean $\pm S E(n=3)$. (A) pH effect on enzymatic activity, a different buffer, was a function of $\mathrm{pH}$ evaluated as described in the Materials and Methods section. (B) activity profile at different temperatures. Cell holder temperature within the reaction cell was stabilized by $60-\mathrm{sec}$ min each assay. (C) The plot of linearized Arrhenius equation, a temperature in which enzymatic activity starts decreasing (inflection point), was fitted to a linear model $\left(R^{2}=0.985\right)$. InK, the natural logarithm of initial velocities; temperatures were in Kelvin degrees. (D) Thermal stability of $\mathrm{VpTLH}$, data were fitted to the Boltzmann sigmoidal model $\left(R^{2}=0.99\right)$. All data were analyzed in Prism5 Graphpad ${ }^{\circledR}$ program. 

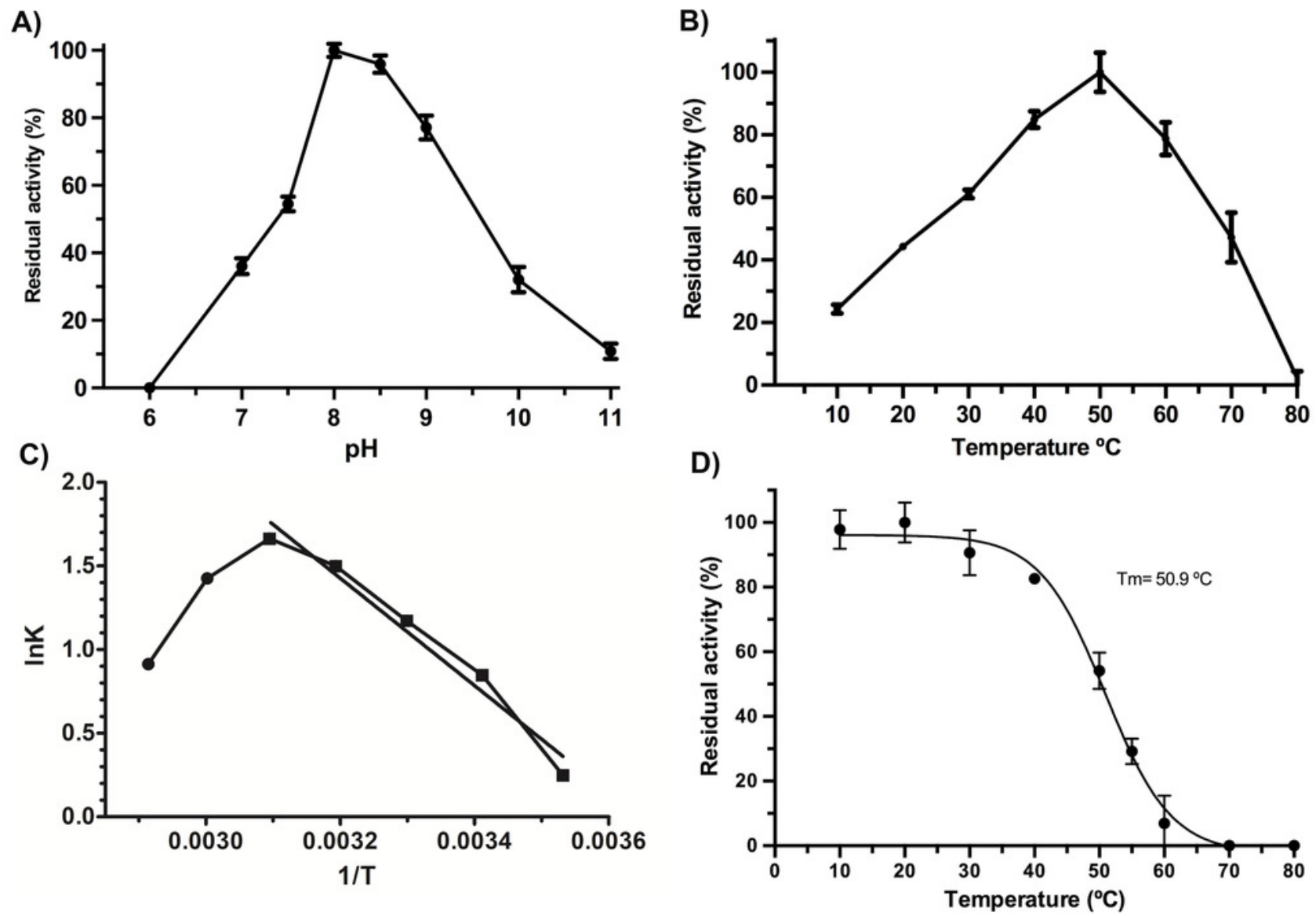
Figure 3

Effect of substrate concentration (PNPL) on VpTLH enzymatic activity.

Fitting data calculated Michaelis-Menten kinetics parameters to non-linear regression model $\left(R^{2}=0.9851\right)$. All substrate concentrations were assayed by triplicate.

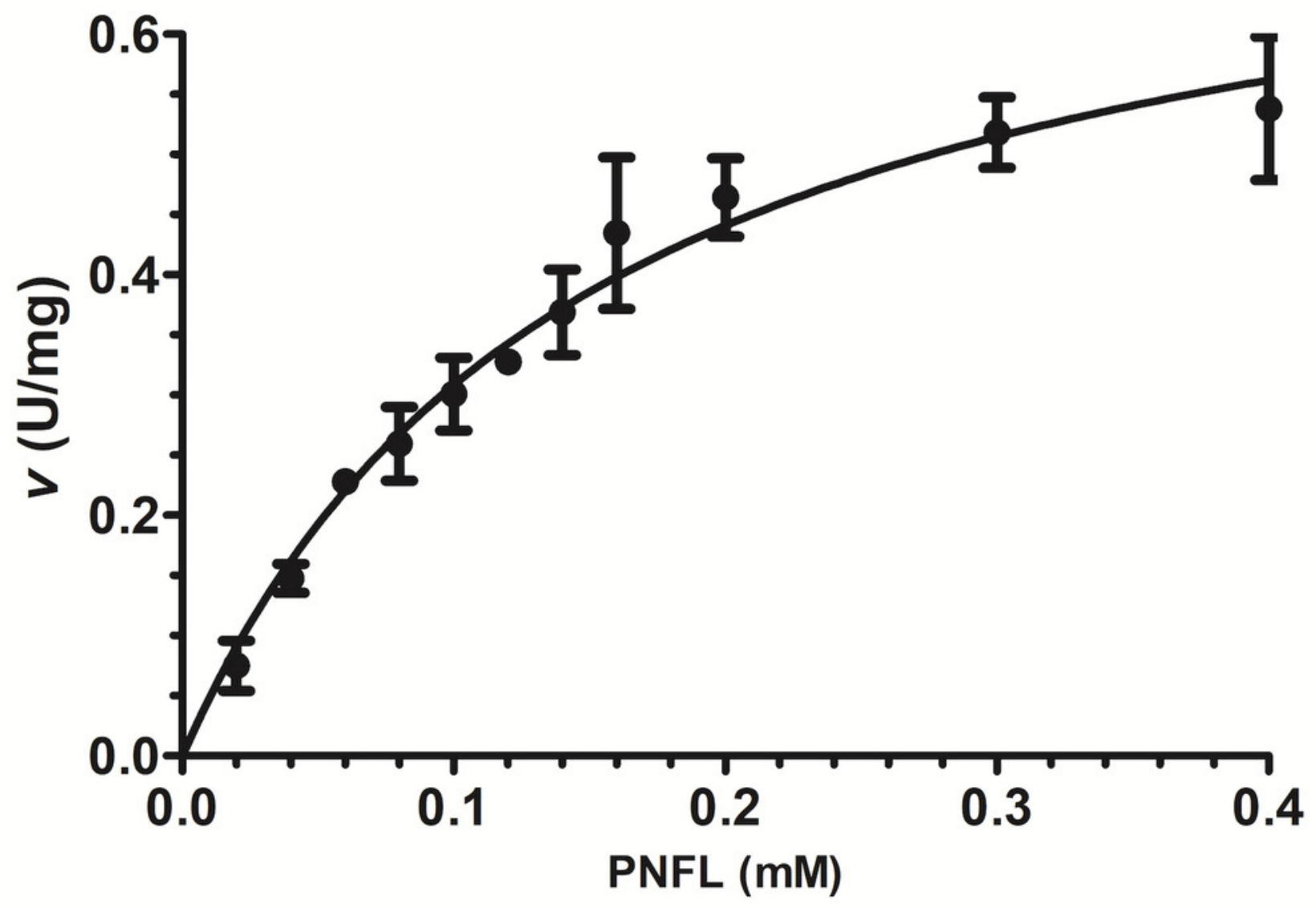




\section{Figure 4}

Effect of phenolic acids on VpTLH enzymatic activity.

VpTLH activity was assayed in the presence of each phenolic acid and the final concentration as indicated. Residual activity was calculated based on VpTLH activity under optimal assay conditions in the absence of phenolics acids. Results are mean SE $(n=3)$ statistical differences $(p<0.05)$ compared to control without phenolics acids as denoted with an asterisk. Control (-), VpTLH without phenolic acids; GA, gallic acid; PR, protocatechuic acid; $\mathrm{CL}$, chlorogenic acid and $\mathrm{VA}$, vanillic acid. 


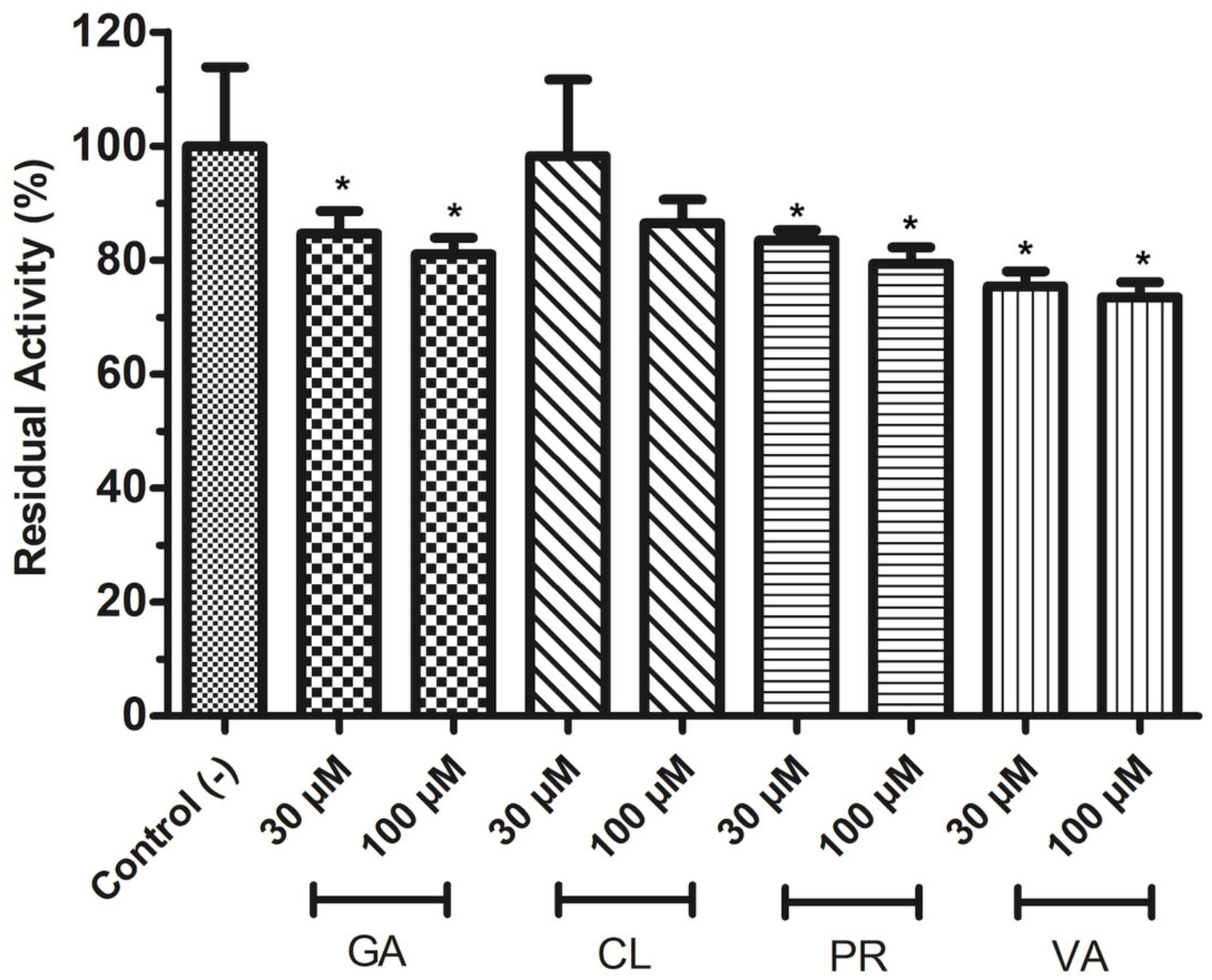


Figure 5

Dose-response analysis of VPTLH inhibition by flavonoids.

VpTLH enzymatic activity was assayed $(n=3)$ in presence of each flavonoid; data were fitted $\left(R^{2}>0.95\right)$ to the dose-response model to calculate IC50 values. Residual activity was calculated as a percentage considering VpTLH enzymatic activity in the absence of tested flavonoids as $100 \% . E G C G=$ Epigallocatechingallate

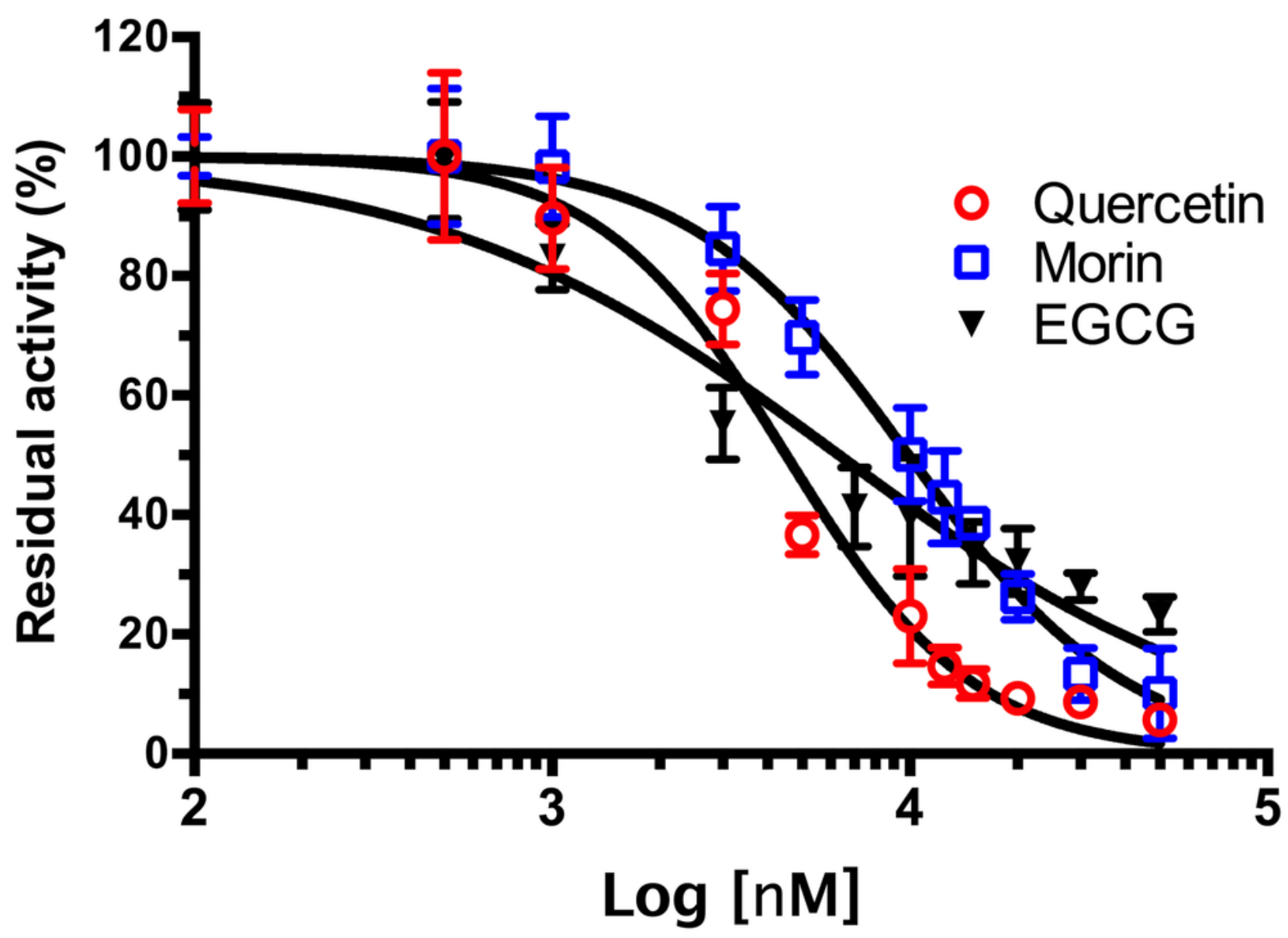


Figure 6

Inhibition of VpTLH hemolytic activity by flavonoids.

Each inhibitor concentration was assayed in triplicate. Bars represented SEM. Hemolytic activity in the presence of flavonoids was calculated as percentage respect to VpTLH without inhibitors (CN). Inhibitor concentrations with statically significant differences $(p<0.05)$ compared to control are denoted with an asterisk.

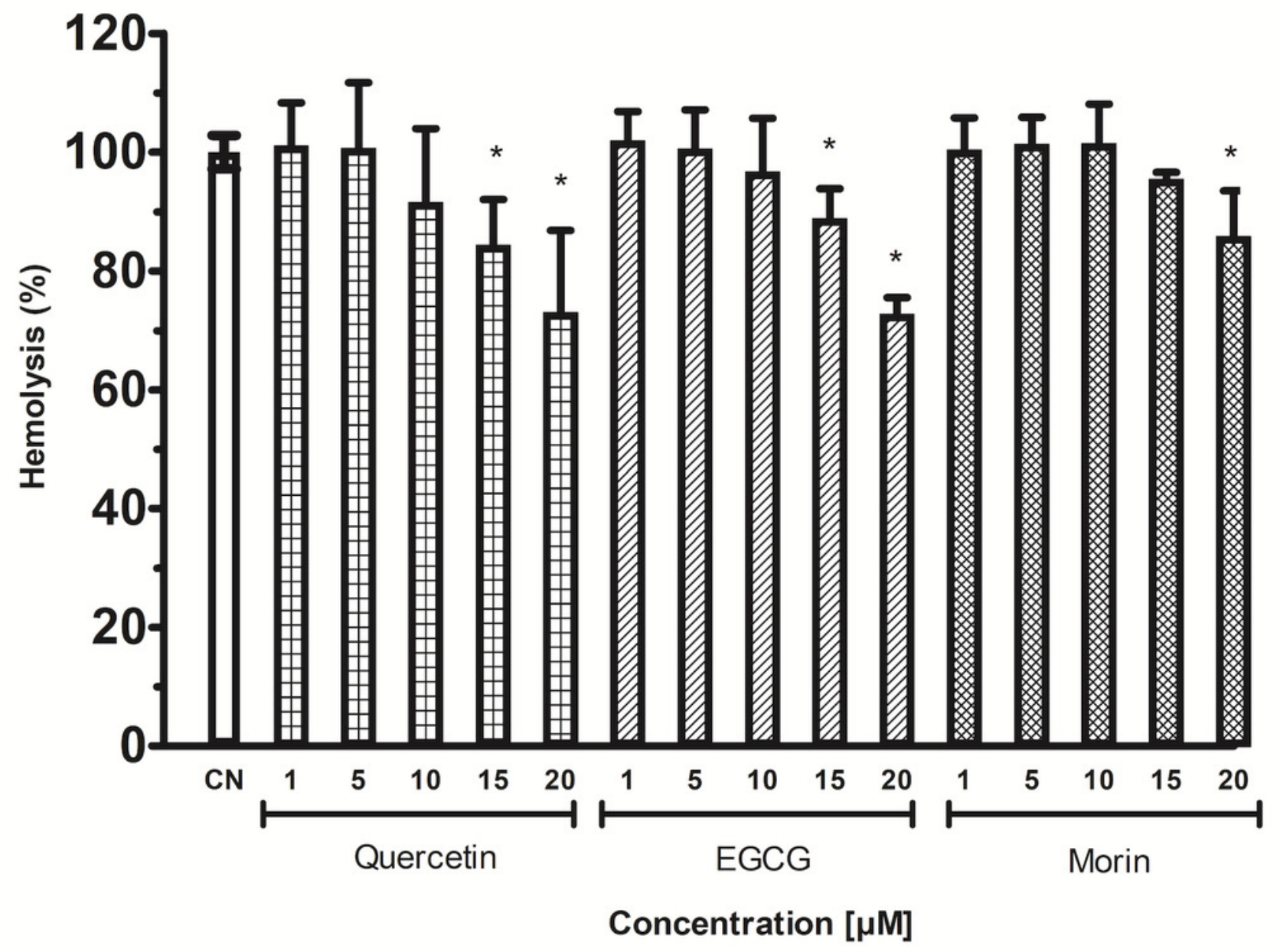


Figure 7

Predicted Structure of the VpTLH.

A) Overall structure superposition of the VpTLH and the VvTLH. N- and C-terminal domains are showed by magenta/cyan $(V p)$ and gray/orange $(V v)$. The cylinders colored by atom type shows the catalytic triad (Ser-His-Asp). (B) Superposition of the catalytic amino acids VpTLH (carbon atoms in gray) and Vv (carbon atoms are purple). (C) Hydrogen bonds (continuous lines) interactions in catalytic amino acids of VpTLH. (D) Charge surface representationVpTLH catalytic site cavity (indicated by the arrow).

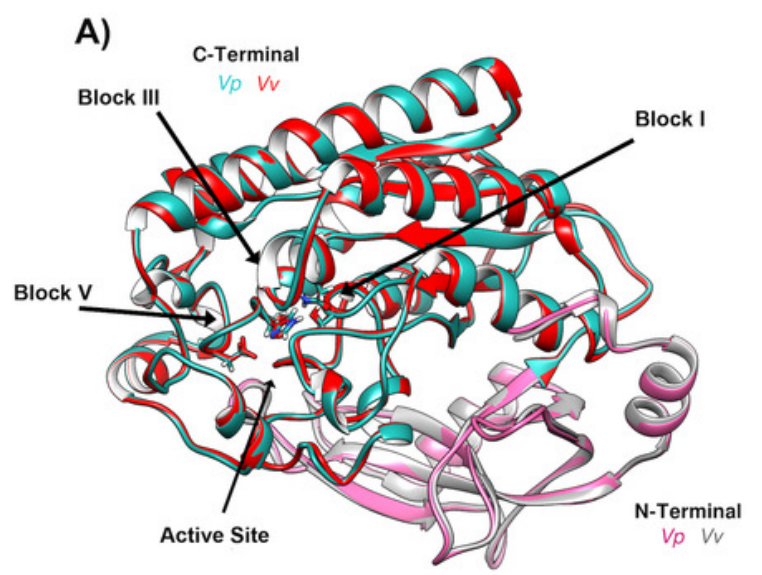

C)

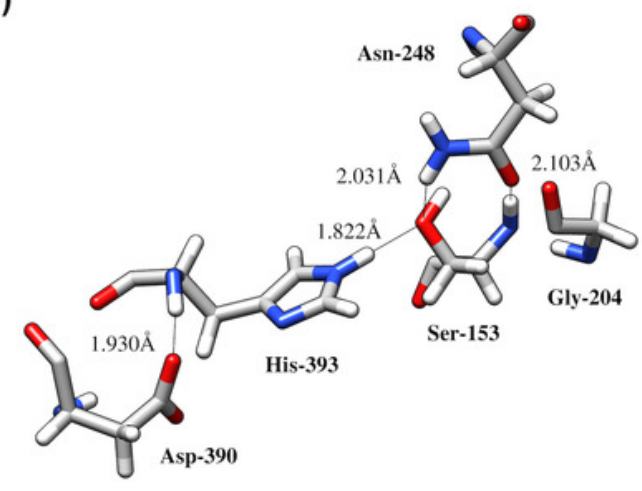

B)

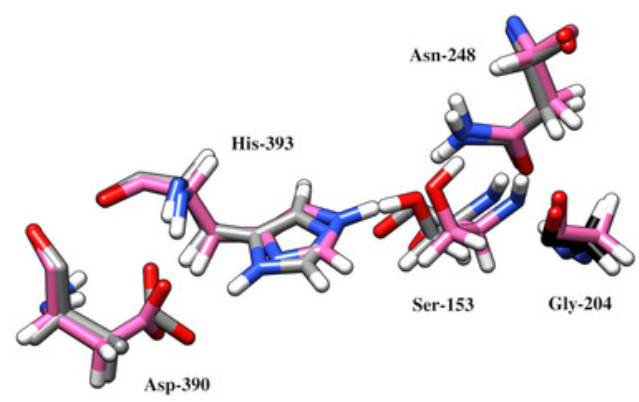

D)

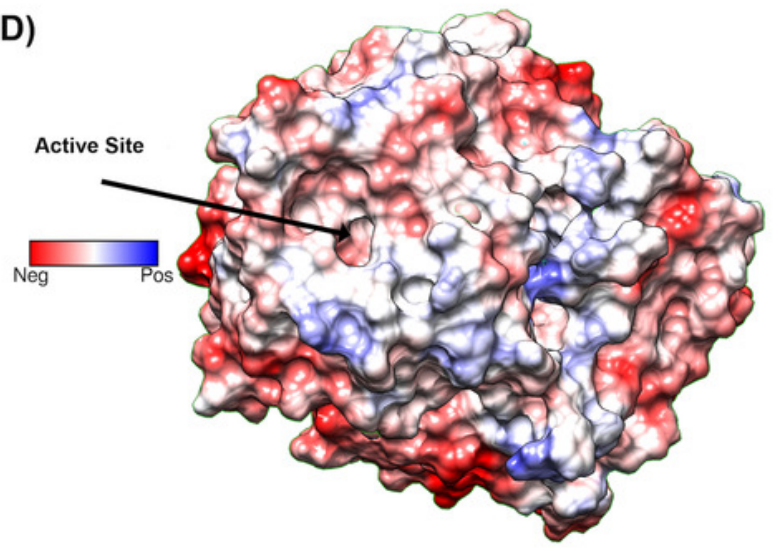




\section{Figure 8}

Molecular docking (A) and interaction maps (B) of substrates into VpTLH active site.

PC, phosphatidylcholine and PNPL, p-nitrophenylaurate. The protein molecule is displayed as a surface in white and ligand as a cylinder colored by atom type with carbon atoms in green. Interaction maps were showed depicted by color as follows: hydrogen bonds (green), alkyl (pink), saline bridge (orange), and Van der Waals interactions (light green). 


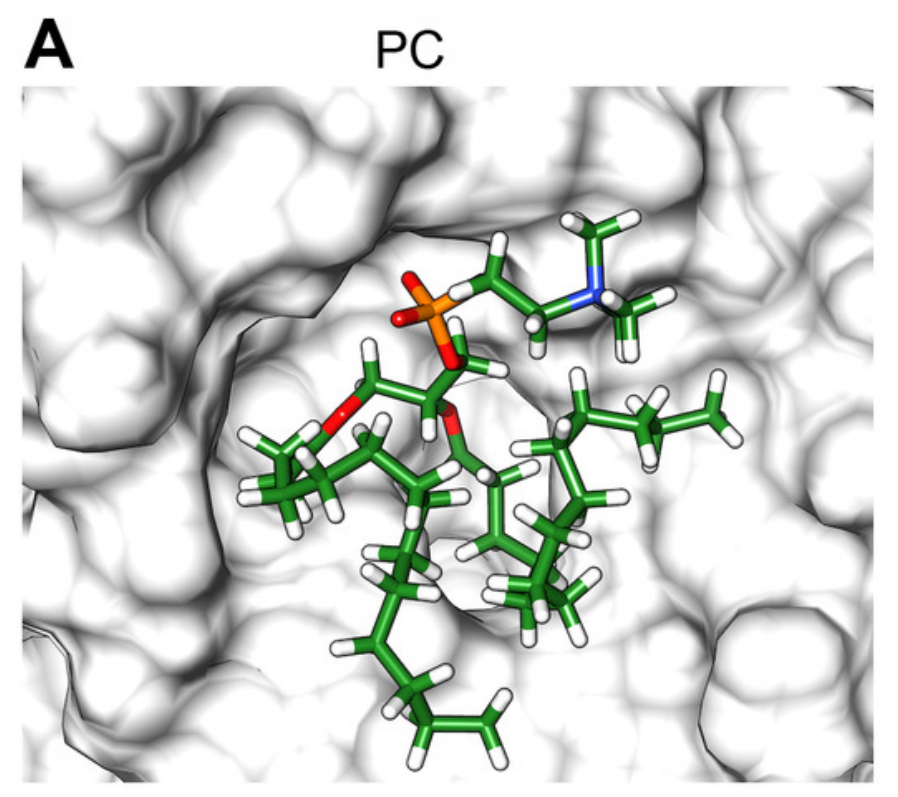

PNPL

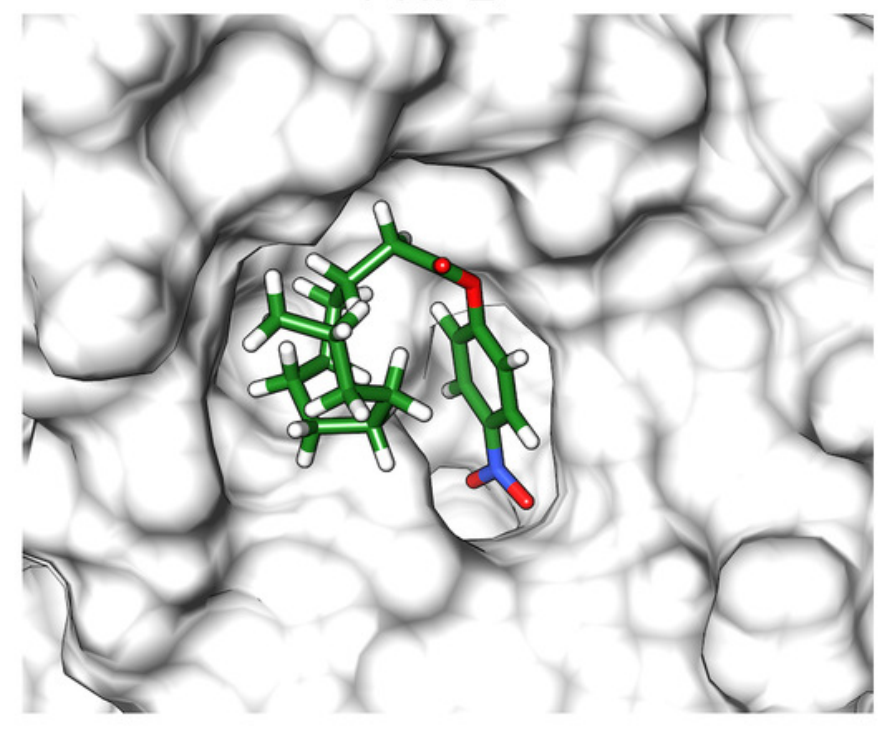

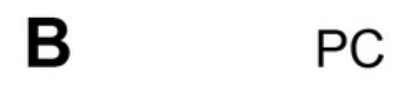

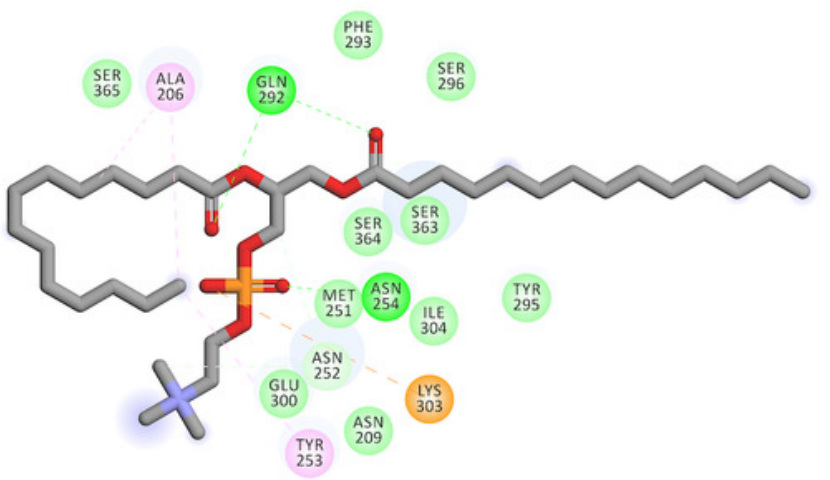

PNPL

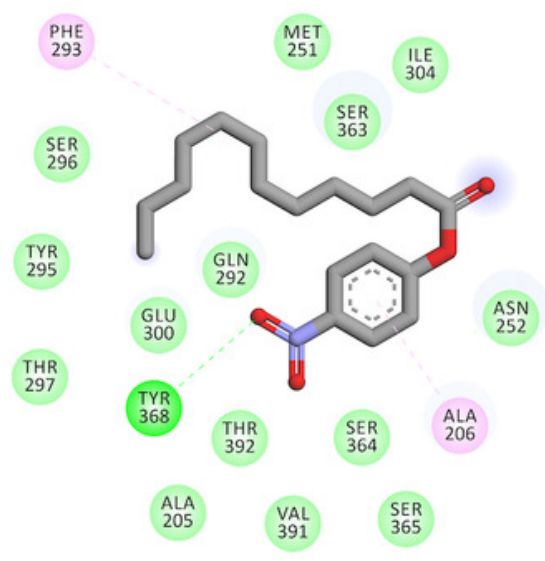




\section{Figure 9}

Molecular docking (A) and interaction map (B) of flavonoids into the VpTLH active site.

EGCG = epigallocatechin gallate. The protein molecule is displayed as a surface in white and ligand as a cylinder colored by atom type with carbon atoms in green. Interaction maps were depicted by color as follows: hydrogen bonds (green), - -alkyl (pink), "-anion (orange), unfavorable donor/acceptor hydrogen bond (red), and Van der Waals interaction (light green). 

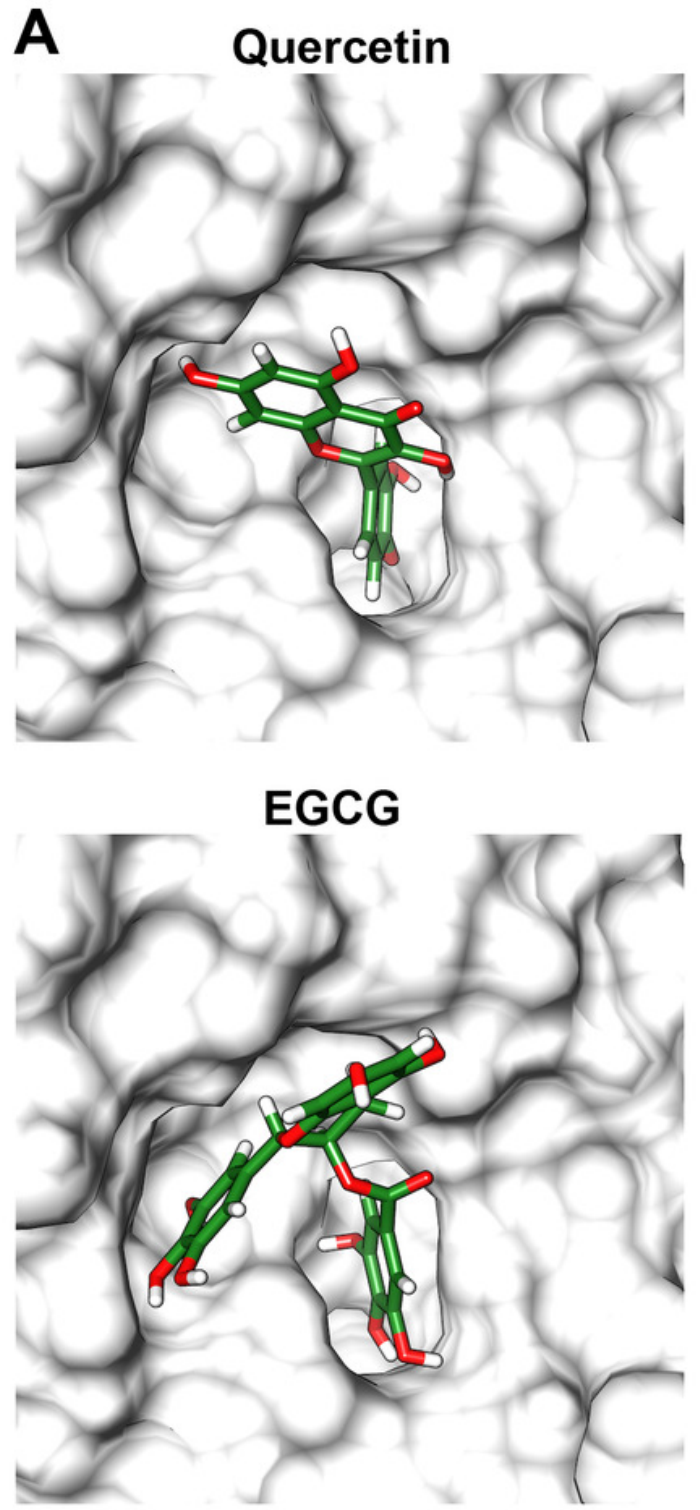

Morin

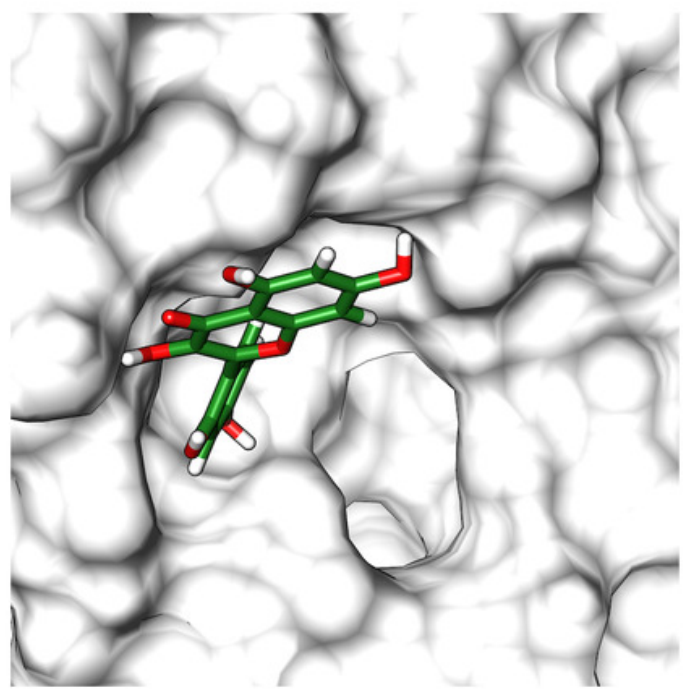

B

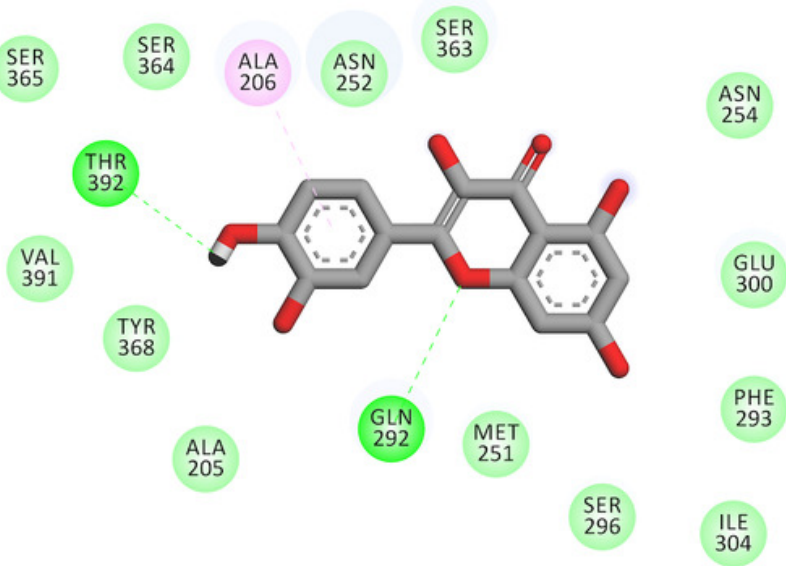

EGCG

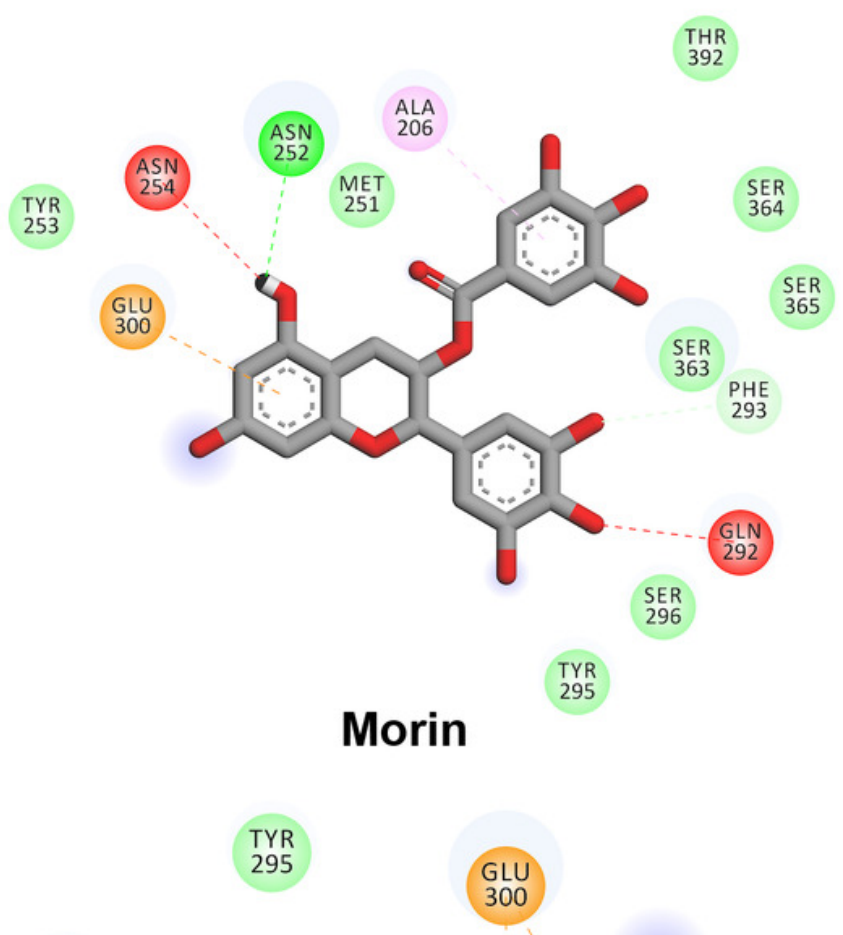

SER
363

GLN

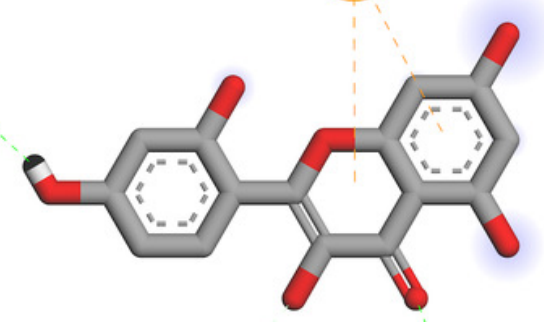

PHE
293

SER

THR 